\title{
BI-EXACT GROUPS, STRONGLY ERGODIC ACTIONS AND GROUP MEASURE SPACE TYPE III FACTORS WITH NO CENTRAL SEQUENCE
}

\author{
CYRIL HOUDAYER AND YUSUKE ISONO
}

\begin{abstract}
We investigate the asymptotic structure of (possibly type III) crossed product von Neumann algebras $M=B \rtimes \Gamma$ arising from arbitrary actions $\Gamma \curvearrowright B$ of bi-exact discrete groups (e.g. free groups) on amenable von Neumann algebras. We prove a spectral gap rigidity result for the central sequence algebra $N^{\prime} \cap M^{\omega}$ of any nonamenable von Neumann subalgebra with normal expectation $N \subset M$. We use this result to show that for any strongly ergodic essentially free nonsingular action $\Gamma \curvearrowright(X, \mu)$ of any bi-exact countable discrete group on a standard probability space, the corresponding group measure space factor $\mathrm{L}^{\infty}(X) \rtimes \Gamma$ has no nontrivial central sequence. Using recent results of Boutonnet-Ioana-Salehi Golsefidy [BISG15], we construct, for every $0<\lambda \leq 1$, a type $\mathrm{III}_{\lambda}$ strongly ergodic essentially free nonsingular action $\mathbf{F}_{\infty} \curvearrowright\left(X_{\lambda}, \mu_{\lambda}\right)$ of the free group $\mathbf{F}_{\infty}$ on a standard probability space so that the corresponding group measure space type $\mathrm{III}_{\lambda}$ factor $\mathrm{L}^{\infty}\left(X_{\lambda}, \mu_{\lambda}\right) \rtimes \mathbf{F}_{\infty}$ has no nontrivial central sequence by our main result. In particular, we obtain the first examples of group measure space type III factors with no nontrivial central sequence.
\end{abstract}

\section{Introduction And Statement of the MAin RESUlts}

The group measure space construction of Murray and von Neumann [MvN43] associates to any ergodic (essentially) free nonsingular action $\Gamma \curvearrowright(X, \mu)$ of a countable discrete group on a standard probability space a factor denoted by $\mathrm{L}^{\infty}(X) \rtimes \Gamma$. A fundamental question in operator algebras is how much information does the group measure space factor $\mathrm{L}^{\infty}(X) \rtimes \Gamma$ retain from the group action $\Gamma \curvearrowright(X, \mu)$ ? This question has attracted a lot of attention during the last 15 years and several important developments regarding the structure and the rigidity of group measure space factors have been made possible thanks to Popa's deformation/rigidity theory [P006a]. We refer the reader to Ga10, Va10, Io12b] for recent surveys on this topic.

One of the questions we address in this paper is the following general problem: Under which assumptions on the countable discrete group $\Gamma$ and the ergodic free nonsingular action $\Gamma \curvearrowright$ $(X, \mu)$, the group measure space factor $\mathrm{L}^{\infty}(X) \rtimes \Gamma$ is full? Recall from [Co74] that a factor $M$ with separable predual is full if its asymptotic centralizer $M_{\omega}$ is trivial for some (or any) nonprincipal ultrafilter $\omega \in \beta(\mathbf{N}) \backslash \mathbf{N}$. By [AH12, Theorem 5.2], a factor $M$ with separable predual is full if and only if its central sequence algebra $M^{\prime} \cap M^{\omega}$ is trivial for some (or any) nonprincipal ultrafilter $\omega \in \beta(\mathbf{N}) \backslash \mathbf{N}$ (see Section 2 for further details). If the group measure space factor $\mathrm{L}^{\infty}(X) \rtimes \Gamma$ is full then the free nonsingular action $\Gamma \curvearrowright(X, \mu)$ is necessarily strongly ergodic, that is, any $\Gamma$-asymptotically invariant sequence of measurable subsets of $X$ is trivial. The converse is not true in general as demonstrated in the celebrated example by Connes and Jones [J 81]. Indeed, they exhibited an example of a strongly ergodic free probability measure preserving (pmp) action such the associated group measure space $\mathrm{II}_{1}$ factor is $M c D$ uff, that is, tensorially absorbs the hyperfinite $\mathrm{II}_{1}$ factor of Murray and von Neumann.

2010 Mathematics Subject Classification. 46L10, 46L36, 46L06, 37A20.

Key words and phrases. Bi-exact discrete groups; Full factors; Group measure space construction; Ozawa's condition (AO); Popa's intertwining techniques; Strongly ergodic actions; Ultraproduct von Neumann algebras.

$\mathrm{CH}$ is supported by ERC Starting Grant GAN 637601.

YI is supported by JSPS Research Fellowship. 
The general problem mentioned above has nevertheless a satisfactory answer in the case when the action $\Gamma \curvearrowright(X, \mu)$ is pmp. Indeed, it was shown by Choda in Ch81 that when the countable discrete group $\Gamma$ is not inner amenable and the free pmp action $\Gamma \curvearrowright(X, \mu)$ is strongly ergodic, then the group measure space $\mathrm{II}_{1}$ factor $\mathrm{L}^{\infty}(X) \rtimes \Gamma$ is full. The facts that the group $\Gamma$ is not inner amenable and the action $\Gamma \curvearrowright(X, \mu)$ is pmp imply that all the central sequences in $\mathrm{L}^{\infty}(X) \rtimes \Gamma$ must asymptotically lie in $\mathrm{L}^{\infty}(X)$. It follows immediately that $\mathrm{L}^{\infty}(X) \rtimes \Gamma$ is full if the action is strongly ergodic. In the above reasoning, the assumption that the action $\Gamma \curvearrowright(X, \mu)$ is pmp is crucial since nonamenable (and in particular non-inner amenable) groups always admit an amenable (in the sense of Zimmer [Zi84, Definition 4.3.1]) type III ergodic nonsingular action, namely the Poisson boundary action. Very little is known about the general problem mentioned above when the action $\Gamma \curvearrowright(X, \mu)$ is no longer pmp and is more generally nonsingular (possibly of type III).

In this paper, we investigate the asymptotic structure of (possibly type III) group measure space factors $\mathrm{L}^{\infty}(X) \rtimes \Gamma$ and more generally of (possibly type III) crossed product von Neumann algebras $B \rtimes \Gamma$ arising from arbitrary actions $\Gamma \curvearrowright B$ of bi-exact discrete groups on amenable von Neumann algebras. The class of bi-exact discrete groups was introduced by Ozawa in Oz04] (see also [BO08, Chapter 15]) and includes amenable groups, free groups, Gromov word-hyperbolic groups and discrete subgroups of connected simple Lie groups of real rank one. We refer the reader to Section 2 for a precise definition. Any bi-exact discrete group is either amenable or non-inner amenable Oz04]. Ozawa's celebrated result Oz03 asserts that bi-exact discrete groups $\Gamma$ give rise to solid group von Neumann algebras $\mathrm{L}(\Gamma)$, that is, for any diffuse von Neumann algebra $A \subset \mathrm{L}(\Gamma)$, the relative commutant $A^{\prime} \cap \mathrm{L}(\Gamma)$ is amenable. Moreover, any solid $\mathrm{II}_{1}$ factor is either amenable or full $\mathrm{Oz03}$, Proposition 7]. Recall that an inclusion of von Neumann algebras $N \subset M$ is with expectation if there exists a faithful normal conditional expectation $\mathrm{E}_{N}: M \rightarrow N$.

Our first main result is a spectral gap rigidity result inside crossed product von Neumann algebras $M=B \rtimes \Gamma$ arising from arbitrary actions $\Gamma \curvearrowright B$ of bi-exact discrete groups on amenable $\sigma$-finite von Neumann algebras. More precisely, we prove that for any von Neumann subalgebra with expectation $N \subset M$, either $N$ has a nonzero amenable direct summand or the central sequence algebra $N^{\prime} \cap M^{\omega}$ lies in the smaller algebra $B^{\omega} \rtimes \Gamma$. Our Theorem $\mathrm{A}$ can be regarded as an analogue of the spectral gap rigidity results discovered by Peterson in $\mathrm{Pe} 06$, Theorem 4.3] and Popa in [Po06b, Theorem 1.5] and [Po06c, Lemma 2.2].

Theorem A. Let $\Gamma$ be any bi-exact discrete group, $B$ any amenable $\sigma$-finite von Neumann algebra and $\Gamma \curvearrowright B$ any action. Denote by $M:=B \rtimes \Gamma$ the corresponding crossed product von Neumann algebra. Let $p \in M$ be any nonzero projection and $N \subset p M p$ any von Neumann subalgebra with expectation. Let $\omega \in \beta(\mathbf{N}) \backslash \mathbf{N}$ be any nonprincipal ultrafilter.

Then at least one of the following conditions holds true:

- The von Neumann algebra $N$ has a nonzero amenable direct summand.

- We have $N^{\prime} \cap p M^{\omega} p \subset p\left(B^{\omega} \rtimes \Gamma\right) p$. In this case, we further obtain $A \preceq_{B^{\omega} \rtimes \Gamma} B^{\omega}$ for any finite von Neumann subalgebra with expectation $A \subset N^{\prime} \cap p M^{\omega} p$.

We refer the reader to Section 2 for ultraproduct von Neumann algebras and Popa's intertwining techniques inside arbitrary von Neumann algebras. The proof of Theorem A given in Section 44 (see Theorems 4.1 and 4.2) uses a combination of Ozawa's $\mathrm{C}^{*}$-algebraic techniques Oz03, Oz04, Is12, ultraproduct von Neumann algebraic techniques Oc85, AH12 and the recent generalization of Popa's intertwining-by-bimodules to the framework of type III von Neumann algebras developed by the authors in [HI15. The interesting feature of the proof of Theorem $\mathrm{A}$ is that it does not rely on Connes-Tomita-Takesaki modular theory. Indeed, unlike other instances of Popa's spectral gap rigidity results in the literature which typically rely on using amenable traces and hence require the ambient von Neumann algebra to be (semi)finite, we 
use instead unital completely positive (ucp) maps and exploit Ozawa's $\mathrm{C}^{*}$-algebraic techniques Oz03, Oz04 to prove the existence of norm one projections. The main advantage of this approach is that it allows us to work directly inside the (possibly type III) crossed product von Neumann algebra $M=B \rtimes \Gamma$ without appealing to the continuous core decomposition. In this respect, our approach is similar to the one we developed in our previous paper [HI15]. We refer the reader to [HR14, HU15, HV12, Is12, Is13, for other structural/rigidity results for type III factors involving the continuous core decomposition.

Following [HR14, Oz04], we say that a von Neumann algebra $M$ is $\omega$-semisolid if for any von Neumann subalgebra $N \subset M$ with expectation such that the relative commutant $N^{\prime} \cap M^{\omega}$ has no type I direct summand, we have that $N$ is amenable. The next corollary strengthens the indecomposability properties of crossed product von Neumann algebras $B \rtimes \Gamma$ arising from arbitrary actions $\Gamma \curvearrowright B$ of bi-exact discrete groups on abelian von Neumann algebras (see Oz04, HV12, Is12 for previous results).

Corollary B. Let $\Gamma$ be any bi-exact discrete group, $B$ any abelian $\sigma$-finite von Neumann algebra and $\Gamma \curvearrowright B$ any action. Let $\omega \in \beta(\mathbf{N}) \backslash \mathbf{N}$ be any nonprincipal ultrafilter. Then the crossed product von Neumann algebra $B \rtimes \Gamma$ is $\omega$-semisolid.

In particular, if $B \rtimes \Gamma$ is a nonamenable factor, then $B \rtimes \Gamma$ is prime, that is, $B \rtimes \Gamma$ cannot be written as a tensor product $Q_{1} \bar{\otimes} Q_{2}$ of diffuse factors.

Our second main result, Theorem $\mathrm{C}$ below, is an answer to the general problem mentioned earlier in the case when the acting group is bi-exact. Indeed, using Theorem $\mathrm{A}$ in the case when the action $\Gamma \curvearrowright B$ arises from a strongly ergodic free nonsingular action $\Gamma \curvearrowright(X, \mu)$ of a bi-exact countable discrete group on a standard probability space, we show that the group measure space factor $\mathrm{L}^{\infty}(X) \rtimes \Gamma$ is full.

Theorem C. Let $\Gamma$ be any bi-exact countable discrete group and $\Gamma \curvearrowright(X, \mu)$ any strongly ergodic free nonsingular action on a standard probability space. Then the group measure space factor $\mathrm{L}^{\infty}(X) \rtimes \Gamma$ is full.

The proof of Theorem $\mathrm{C}$ uses a combination of Theorem $\mathrm{A}$ and the useful Lemma 5.1 below which proves the existence of a nontrivial centralizing sequence $\left(u_{n}\right)_{n}$ in every nonfull factor $M=\mathrm{L}(\mathcal{R})$ arising from a strongly ergodic nonsingular equivalence relation $\mathcal{R}$ defined on a standard probability space such that $\left(u_{n}\right)_{n}$ "does not embed" into the Cartan subalgebra $\mathrm{L}^{\infty}(X)$. Our Lemma 5.1 is a nonsingular generalization of a recent result of Hoff (see the first part of the proof of [Ho15, Proposition C]). In view of Choda's result [Ch81, we do not know whether Theorem C holds true more generally for (arbitrary strongly ergodic free nonsingular actions of) arbitrary non-inner amenable groups instead of bi-exact groups. We point out that Ozawa recently showed in Oz16 that Theorem Cholds true for arbitrary strongly ergodic free nonsingular actions of $\mathrm{SL}_{3}(\mathbf{Z})$, which is not bi-exact by [Sa09].

We finally exploit recent results of Boutonnet-Ioana-Salehi Golsefidy [BISG15] to construct, for every $0<\lambda \leq 1$, examples of type $\mathrm{III}_{\lambda}$ strongly ergodic free nonsingular actions of a free group on a standard probability space. It is shown in [BISG15, Theorem A] that for any (not necessarily compact) connected simple Lie group and any countable dense subgroup $\Lambda<G$ with "algebraic entries" (e.g. $(\Lambda<G)=\left(\mathrm{SL}_{n}(\mathbf{Q})<\mathrm{SL}_{n}(\mathbf{R})\right)$ for $\left.n \geq 2\right)$, the left translation action $\Lambda \curvearrowright G$ is strongly ergodic. By taking a suitable non-unimodular closed subgroup $P<G$, the quotient action $\Lambda \curvearrowright G / P$ is still strongly ergodic and of type III. The quotient action $\Lambda \curvearrowright G / P$ need not be essentially free in general. However, using a "direct product" construction similar to the one used in [HV12, Corollary B], we can then construct strongly ergodic essentially free nonsingular actions of free groups and we obtain the following corollary. 
Corollary D. For every $0<\lambda \leq 1$, there exists a strongly ergodic free nonsingular action $\mathbf{F}_{\infty} \curvearrowright\left(X_{\lambda}, \mu_{\lambda}\right)$ of type $\mathrm{III}_{\lambda}$ so that the group measure space factor $\mathrm{L}^{\infty}\left(X_{\lambda}, \mu_{\lambda}\right) \rtimes \mathbf{F}_{\infty}$ is of type $\mathrm{III}_{\lambda}$ and is full.

Moreover, there exists a strongly ergodic free nonsingular action $\mathbf{F}_{\infty} \curvearrowright\left(X_{\infty}, \mu_{\infty}\right)$ of type $\mathrm{II}_{\infty}$ so that the group measure space factor $\mathrm{L}^{\infty}\left(X_{\infty}, \mu_{\infty}\right) \rtimes \mathbf{F}_{\infty}$ is of type $\mathrm{II}_{\infty}$ and is full.

The first examples of full factors of type III were discovered by Connes in Co74]. He showed that the factors

$$
M_{n, k, \varphi}=\left(\bar{\bigotimes}_{\mathbf{F}_{n}}\left(\mathbf{M}_{k}(\mathbf{C}), \varphi\right)\right) \rtimes \mathbf{F}_{n}
$$

arising from Connes-Størmer Bernoulli shifts of free groups $\mathbf{F}_{n} \curvearrowright \bar{\bigotimes}_{\mathbf{F}_{n}}\left(\mathbf{M}_{k}(\mathbf{C}), \varphi\right)$ are full if $n, k \geq 2$ and of type III if $\varphi$ is not tracial. Observe that the factors $M_{n, k, \varphi}$ possess $\bar{\bigotimes}_{\mathbf{F}_{n}} \mathbf{C}^{k}$ as a Cartan subalgebra. This implies that the underlying ergodic nonsingular equivalence relation is strongly ergodic [FM75]. However, Connes-Størmer Bernoulli crossed products need not be *-isomorphic to group measure space factors. In this respect, Corollary D above provides the first class of group measure space type III factors with no nontrivial central sequence.

We finally point out that the group measure space type III factors in Corollary D possess a unique Cartan subalgebra, up to unitary conjugacy, by [HV12, Theorem A] (see [PV11] for the trace preserving case). Moreover, Corollary D provides new examples of group measure type III factors with a unique Cartan subalgebra, up to unitary conjugacy. Indeed, the examples of ergodic free nonsingular actions considered in [HV12, Corollary B] are not strongly ergodic since they have an amenable action as a quotient. In particular, the group measure space type III factors in [HV12, Corollary B] are not full while the ones in Corollary D are full.

Acknowledgments. It is our pleasure to thank Adrian Ioana, Dimitri Shlyakhtenko, Yoshimichi Ueda and Stefaan Vaes for their valuable comments.

\section{CONTEnts}

1. Introduction and statement of the main results

2. Preliminaries

3. Bi-exactness and Ozawa's condition (AO)

4. Proofs of Theorem $\mathrm{A}$ and Corollary $\mathrm{B}$

5. Proof of Theorem C

6. Group measure space type III factors with no central sequence

7. Further remarks

References

\section{Preliminaries}

For any von Neumann algebra $M$, we will denote by $\mathcal{Z}(M)$ the centre of $M$, by $\mathcal{U}(M)$ the group of unitaries in $M$ and by $\left(M, \mathrm{~L}^{2}(M), J^{M}, \mathfrak{P}^{M}\right)$ a standard form for $M$. We will say that an inclusion of von Neumann algebras $P \subset 1_{P} M 1_{P}$ is with expectation if there exists a faithful normal conditional expectation $\mathrm{E}_{P}: 1_{P} M 1_{P} \rightarrow P$. 
Crossed product von Neumann algebras. We will use the following terminology and notation regarding crossed product von Neumann algebras. Let $\Gamma$ be any discrete group, $B$ any $\sigma$-finite von Neumann algebra and $\Gamma \curvearrowright B$ any action. Denote by $M:=B \rtimes \Gamma$ the corresponding crossed product von Neumann algebra and by $\mathrm{E}_{B}: M \rightarrow B$ the canonical faithful normal conditional expectation given by $\mathrm{E}_{B}\left(b \lambda_{g}\right)=\delta_{g, e} b$ for all $g \in \Gamma$ and all $b \in B$. Fix a standard form $\left(B, \mathrm{~L}^{2}(B), J^{B}, \mathfrak{P}^{B}\right)$ for $B$. Denote by $u: \Gamma \rightarrow \mathcal{U}\left(\mathrm{L}^{2}(B)\right)$ the canonical unitary representation implementing the action $\Gamma \curvearrowright B$. A standard form $\left(M, \mathrm{~L}^{2}(M), J^{M}, \mathfrak{P}^{M}\right)$ for $M$ is given by $\mathrm{L}^{2}(M)=\mathrm{L}^{2}(B) \otimes \ell^{2}(\Gamma)$ and

$$
J^{M}\left(\xi \otimes \delta_{g}\right)=u_{g}^{*} J^{B} \xi \otimes \delta_{g^{-1}} \quad \text { for all } \xi \in \mathrm{L}^{2}(B) \text { and all } g \in \Gamma .
$$

The Jones projection $e_{B}: \mathrm{L}^{2}(M) \rightarrow \mathrm{L}^{2}(B)$ is then simply given by $e_{B}=1 \otimes P_{\mathbf{C} \delta_{e}}$ where $P_{\mathbf{C} \delta_{e}}: \ell^{2}(\Gamma) \rightarrow \mathbf{C} \delta_{e}$ is the orthogonal projection onto $\mathbf{C} \delta_{e}$. For crossed product von Neumann algebras $M=B \rtimes \Gamma$, we will always use such a standard form $\left(M, \mathrm{~L}^{2}(M), J^{M}, \mathfrak{P}^{M}\right)$ as defined above.

Ultraproduct von Neumann algebras. Let $M$ be any $\sigma$-finite von Neumann algebra and $\omega \in \beta(\mathbf{N}) \backslash \mathbf{N}$ any nonprincipal ultrafilter. Define

$$
\begin{aligned}
\mathcal{I}_{\omega}(M) & =\left\{\left(x_{n}\right)_{n} \in \ell^{\infty}(M) \mid x_{n} \rightarrow 0 * \text {-strongly as } n \rightarrow \omega\right\} \\
\mathcal{M}^{\omega}(M) & =\left\{\left(x_{n}\right)_{n} \in \ell^{\infty}(M) \mid\left(x_{n}\right)_{n} \mathcal{I}_{\omega}(M) \subset \mathcal{I}_{\omega}(M) \text { and } \mathcal{I}_{\omega}(M)\left(x_{n}\right)_{n} \subset \mathcal{I}_{\omega}(M)\right\} .
\end{aligned}
$$

The multiplier algebra $\mathcal{M}^{\omega}(M)$ is a $\mathrm{C}^{*}$-algebra and $\mathcal{I}_{\omega}(M) \subset \mathcal{M}^{\omega}(M)$ is a norm closed twosided ideal. Following [0c85, §5.1], we define the ultraproduct von Neumann algebra $M^{\omega}$ by $M^{\omega}:=\mathcal{M}^{\omega}(M) / \mathcal{I}_{\omega}(M)$, which is indeed known to be a von Neumann algebra. We denote the image of $\left(x_{n}\right)_{n} \in \mathcal{M}^{\omega}(M)$ by $\left(x_{n}\right)^{\omega} \in M^{\omega}$.

For every $x \in M$, the constant sequence $(x)_{n}$ lies in the multiplier algebra $\mathcal{M}^{\omega}(M)$. We will then identify $M$ with $\left(M+\mathcal{I}_{\omega}(M)\right) / \mathcal{I}_{\omega}(M)$ and regard $M \subset M^{\omega}$ as a von Neumann subalgebra. The map $\mathrm{E}_{\omega}: M^{\omega} \rightarrow M:\left(x_{n}\right)^{\omega} \mapsto \sigma$-weak $\lim _{n \rightarrow \omega} x_{n}$ is a faithful normal conditional expectation. For every faithful state $\varphi \in M_{*}$, the formula $\varphi^{\omega}:=\varphi \circ \mathrm{E}_{\omega}$ defines a faithful normal state on $M^{\omega}$. Observe that $\varphi^{\omega}\left(\left(x_{n}\right)^{\omega}\right)=\lim _{n \rightarrow \omega} \varphi\left(x_{n}\right)$ for all $\left(x_{n}\right)^{\omega} \in M^{\omega}$.

Following [Co74, §2], we define

$$
\mathcal{M}_{\omega}(M):=\left\{\left(x_{n}\right)_{n} \in \ell^{\infty}(M) \mid \lim _{n \rightarrow \omega}\left\|x_{n} \varphi-\varphi x_{n}\right\|=0, \forall \varphi \in M_{*}\right\} .
$$

We have $\mathcal{I}_{\omega}(M) \subset \mathcal{M}_{\omega}(M) \subset \mathcal{M}^{\omega}(M)$. The asymptotic centralizer is defined by $M_{\omega}:=$ $\mathcal{M}_{\omega}(M) / \mathcal{I}_{\omega}(M)$. We have $M_{\omega} \subset M^{\omega}$. Moreover, by [Co74, Proposition 2.8] (see also [AH12, Proposition 4.35]), we have $M_{\omega}=M^{\prime} \cap\left(M^{\omega}\right)^{\varphi^{\omega}}$ for every faithful state $\varphi \in M_{*}$.

Let $Q \subset M$ be any von Neumann subalgebra with faithful normal conditional expectation $\mathrm{E}_{Q}: M \rightarrow Q$. Choose a faithful state $\varphi \in M_{*}$ in such a way that $\varphi=\varphi \circ \mathrm{E}_{Q}$. We have $\ell^{\infty}(Q) \subset$ $\ell^{\infty}(M), \mathcal{I}_{\omega}(Q) \subset \mathcal{I}_{\omega}(M)$ and $\mathcal{M}^{\omega}(Q) \subset \mathcal{M}^{\omega}(M)$. We will then identify $Q^{\omega}=\mathcal{M}^{\omega}(Q) / \mathcal{I}_{\omega}(Q)$ with $\left(\mathcal{M}^{\omega}(Q)+\mathcal{I}_{\omega}(M)\right) / \mathcal{I}_{\omega}(M)$ and be able to regard $Q^{\omega} \subset M^{\omega}$ as a von Neumann subalgebra. Observe that the norm $\|\cdot\|_{\left(\left.\varphi\right|_{Q}\right)^{\omega}}$ on $Q^{\omega}$ is the restriction of the norm $\|\cdot\|_{\varphi^{\omega}}$ to $Q^{\omega}$. Observe moreover that $\left(\mathrm{E}_{Q}\left(x_{n}\right)\right)_{n} \in \mathcal{I}_{\omega}(Q)$ for all $\left(x_{n}\right)_{n} \in \mathcal{I}_{\omega}(M)$ and $\left(\mathrm{E}_{Q}\left(x_{n}\right)\right)_{n} \in \mathcal{M}^{\omega}(Q)$ for all $\left(x_{n}\right)_{n} \in \mathcal{M}^{\omega}(M)$. Therefore, the mapping $\mathrm{E}_{Q^{\omega}}: M^{\omega} \rightarrow Q^{\omega}:\left(x_{n}\right)^{\omega} \mapsto\left(\mathrm{E}_{Q}\left(x_{n}\right)\right)^{\omega}$ is a welldefined conditional expectation satisfying $\varphi^{\omega} \circ \mathrm{E}_{Q^{\omega}}=\varphi^{\omega}$. Hence, $\mathrm{E}_{Q^{\omega}}: M^{\omega} \rightarrow Q^{\omega}$ is a faithful normal conditional expectation. For more on ultraproduct von Neumann algebras, we refer the reader to [AH12, Oc85].

We record the following observation that will be used throughout. Let $\Gamma$ be any discrete group, $B$ any $\sigma$-finite von Neumann algebra and $\Gamma \curvearrowright B$ any action. Put $M:=B \rtimes \Gamma$ and denote by $\mathrm{E}_{B}: M \rightarrow B$ the canonical faithful normal conditional expectation. Choose any faithful state $\varphi \in M_{*}$ such that $\varphi \circ \mathrm{E}_{B}=\varphi$. Then the von Neumann subalgebra $B^{\omega} \vee M \subset M^{\omega}$ is globally invariant under the modular automorphism group $\sigma^{\varphi^{\omega}}$ and hence is with expectation. 
Observe that we have $B^{\omega} \vee M=B^{\omega} \rtimes \Gamma$ canonically. Therefore the von Neumann subalgebra $B^{\omega} \rtimes \Gamma \subset M^{\omega}$ is with expectation. Denote by $\mathrm{E}_{B^{\omega}}: M^{\omega} \rightarrow B^{\omega}$ and by $\mathrm{E}_{B^{\omega} \rtimes \Gamma}: M^{\omega} \rightarrow$ $B^{\omega} \rtimes \Gamma$ the unique $\varphi^{\omega}$-preserving conditional expectations. By uniqueness of the $\varphi^{\omega}$-preserving conditional expectation $\mathrm{E}_{B^{\omega}}: M^{\omega} \rightarrow B^{\omega}$, we have $\mathrm{E}_{B^{\omega}} \circ \mathrm{E}_{B^{\omega} \rtimes \Gamma}=\mathrm{E}_{B^{\omega}}$.

We thank Hiroshi Ando for pointing out to us the following well-known result.

Lemma 2.1. Let $M$ be any $\sigma$-finite von Neumann algebra and $\omega \in \beta(\mathbf{N}) \backslash \mathbf{N}$ any nonprincipal ultrafilter. For any $u \in \mathcal{U}\left(M^{\omega}\right)$, there exists a sequence $\left(u_{n}\right)_{n} \in \mathcal{M}^{\omega}(M)$ such that $u=\left(u_{n}\right)^{\omega}$ and $u_{n} \in \mathcal{U}(M)$ for every $n \in \mathbf{N}$.

Proof. Denote by $f: \mathbf{T} \rightarrow(-\pi, \pi]$ the unique Borel function such that $\exp (\mathrm{i} f(z))=z$ for all $z \in \mathbf{T}$. Let $u \in \mathcal{U}\left(M^{\omega}\right)$ and put $h=f(u) \in M^{\omega}$. Then $h^{*}=h$ and $\exp (\mathrm{i} h)=u$. Write $h=\left(h_{n}\right)^{\omega}$ for some $\left(h_{n}\right)_{n} \in \mathcal{M}^{\omega}(M)$. Since $h^{*}=h$, up to replacing each $h_{n}$ by $\frac{1}{2}\left(h_{n}+h_{n}^{*}\right)$, we may assume that $h_{n}^{*}=h_{n}$ for every $n \in \mathbf{N}$. Put $u_{n}=\exp \left(\mathrm{i} h_{n}\right) \in \mathcal{U}(M)$ for every $n \in \mathbf{N}$. Since $[-\pi, \pi] \rightarrow \mathbf{T}: t \mapsto \exp (\mathrm{i} t)$ is a continuous function, it is a uniform limit of polynomial functions by Stone-Weierstrass theorem. It follows that $\left(u_{n}\right)_{n}=\left(\exp \left(\mathrm{i} h_{n}\right)\right)_{n}=\exp \left(\mathrm{i}\left(h_{n}\right)_{n}\right) \in \mathcal{M}^{\omega}(M)$ and $u=\exp (\mathrm{i} h)=\exp \left(\mathrm{i}\left(h_{n}\right)^{\omega}\right)=\left(\exp \left(\mathrm{i} h_{n}\right)\right)^{\omega}=\left(u_{n}\right)^{\omega}$.

We next recall the construction of the Groh-Raynaud ultraproduct. For any Hilbert space $H$, define the ultraproduct Hilbert space $H_{\omega}$ as the completion/separation of $\ell^{\infty}(H)$ with respect to the semi-inner product given by $\left\langle\left(\xi_{n}\right)_{n},\left(\eta_{n}\right)_{n}\right\rangle:=\lim _{n \rightarrow \omega}\left\langle\xi_{n}, \eta_{n}\right\rangle_{H}$ for all $\left(\xi_{n}\right)_{n},\left(\eta_{n}\right)_{n} \in \ell^{\infty}(H)$. We denote the image of $\left(\xi_{n}\right)_{n} \in \ell^{\infty}(H)$ by $\left(\xi_{n}\right)_{\omega} \in H_{\omega}$. Let $M \subset \mathbf{B}(H)$ be any von Neumann algebra. We define the unital $*$-representation $\pi^{\omega}: \ell^{\infty}(M) \rightarrow \mathbf{B}\left(H_{\omega}\right)$ by

$$
\pi^{\omega}\left(\left(x_{n}\right)_{n}\right)\left(\xi_{n}\right)_{\omega}=\left(x_{n} \xi_{n}\right)_{\omega} \quad \text { for all }\left(x_{n}\right)_{n} \in \ell^{\infty}(\mathbf{B}(H)) \text { and all }\left(\xi_{n}\right)_{n} \in \ell^{\infty}(H) .
$$

Let $\left(M, \mathrm{~L}^{2}(M), J^{M}, \mathfrak{P}^{M}\right)$ be a standard form for $M$. The Groh-Raynaud ultraproduct $N:=$ $\prod^{\omega} M$ is the von Neumann algebra generated by $\pi^{\omega}\left(\ell^{\infty}(M)\right)$. It is known that the inclusion $N \subset \mathbf{B}\left(\mathrm{L}^{2}(M)_{\omega}\right)$ is in standard form with modular conjugation given by $J^{N}\left(\xi_{n}\right)_{\omega}:=\left(J^{M} \xi_{n}\right)_{\omega}$ for all $\left(\xi_{n}\right)_{\omega} \in \mathrm{L}^{2}(M)_{\omega}$ (see [Ra99, Corollary 3.9] and [AH12, Theorem 3.18]). By [AH12, Theorem 3.7], the Ocneanu ultraproduct is *-isomorphic to a corner of the Groh-Raynaud ultraproduct. More precisely, for any faithful state $\varphi \in M_{*}$, denote by $\xi_{\varphi} \in \mathfrak{P}^{M}$ the canonical representing vector. Then the isometry given by

$$
w_{\varphi}: \mathrm{L}^{2}\left(M^{\omega}\right) \rightarrow \mathrm{L}^{2}(M)_{\omega}:\left(x_{n}\right)^{\omega} \xi_{\varphi^{\omega}} \mapsto\left(x_{n} \xi_{\varphi}\right)_{\omega}
$$

satisfies $w_{\varphi}^{*} N w_{\varphi}=M^{\omega}$. Define the ultraproduct state $\varphi_{\omega}=\left\langle\cdot\left(\xi_{\varphi}\right)_{\omega},\left(\xi_{\varphi}\right)_{\omega}\right\rangle \in N_{*}$ and denote by $p \in N$ the support projection of $\varphi_{\omega} \in N_{*}$. We have $w_{\varphi} w_{\varphi}^{*}=p J^{N} p J^{N}$. Then the condition $w_{\varphi}^{*} N w_{\varphi}=M^{\omega}$ implies that $p J^{N} p J^{N} N p J^{N} p J^{N} \cong p N p \cong M^{\omega}$ so that the standard representation of $M^{\omega}$ is given by $\mathrm{L}^{2}\left(M^{\omega}\right)=p J^{N} p J^{N} \mathrm{~L}^{2}(M)_{\omega}$ with modular conjugation $J^{M^{\omega}}=p J^{N} p$.

Lemma 2.2. Let $B \subset M$ be any inclusion of $\sigma$-finite von Neumann algebras with faithful normal conditional expectation $\mathrm{E}_{B}: M \rightarrow B$. Denote by $e_{B}: \mathrm{L}^{2}(M) \rightarrow \mathrm{L}^{2}(B)$ the corresponding Jones projection. Denote by $N:=\prod^{\omega} M$ the Groh-Raynaud ultraproduct. Let $\varphi \in M_{*}$ be any faithful state such that $\varphi \circ \mathrm{E}_{B}=\varphi$ and denote by $p \in N$ the support projection of $\varphi_{\omega} \in N_{*}$.

Then $\pi^{\omega}\left(\left(e_{B}\right)_{n}\right)$ commutes with $p$ and $J^{N}$.

Proof. Since $e_{B}$ commutes with $J^{M}, \pi^{\omega}\left(\left(e_{B}\right)_{n}\right)$ commutes with $J^{N}$. Denote by $\xi_{\varphi} \in \mathfrak{P}^{M}$ the canonical vector representing $\varphi \in M_{*}$. Since $e_{B} M e_{B}=B e_{B}$ and $e_{B} \xi_{\varphi}=\xi_{\varphi}$, we have

$$
\begin{aligned}
\pi^{\omega}\left(\left(e_{B}\right)_{n}\right) J^{N} \pi^{\omega}\left(\ell^{\infty}(M)\right)\left(\xi_{\varphi}\right)_{\omega} & =J^{N} \pi^{\omega}\left(\left(e_{B}\right)_{n}\right) \pi^{\omega}\left(\ell^{\infty}(M)\right) \pi^{\omega}\left(\left(e_{B}\right)_{n}\right)\left(\xi_{\varphi}\right)_{\omega} \\
& =J^{N} \pi^{\omega}\left(\ell^{\infty}(B)\right) \pi^{\omega}\left(\left(e_{B}\right)_{n}\right)\left(\xi_{\varphi}\right)_{\omega} \\
& \subset J^{N} \pi^{\omega}\left(\ell^{\infty}(M)\right)\left(\xi_{\varphi}\right)_{\omega} .
\end{aligned}
$$


Since $p$ is the projection onto the closure of $J^{N} \pi^{\omega}\left(\ell^{\infty}(M)\right)\left(\xi_{\varphi}\right)_{\omega}$, we obtain that $\pi^{\omega}\left(\left(e_{B}\right)_{n}\right)$ commutes with $p$.

\section{Equivalence relations and von Neumann algebras.}

Definition 2.3 ([FM75]). Let $(X, \mu)$ be any standard probability space. A nonsingular equivalence relation $\mathcal{R}$ defined on $(X, \mu)$ is an equivalence relation $\mathcal{R} \subset X \times X$ which satisfies the following three conditions:

(i) $\mathcal{R} \subset X \times X$ is a Borel subset,

(ii) $\mathcal{R}$ has countable classes and

(iii) for every $\varphi \in[\mathcal{R}]$, we have $\left[\varphi_{*} \mu\right]=[\mu]$ where $[\mathcal{R}]$ denotes the full group of $\mathcal{R}$ consisting in all the Borel automorphisms $\varphi: X \rightarrow X$ such that $\operatorname{gr}(\varphi) \subset \mathcal{R}$.

Following [FM75], to any nonsingular equivalence relation $\mathcal{R}$ defined on a standard probability space $(X, \mu)$, one can associate a von Neumann algebra $M=\mathrm{L}(\mathcal{R})$ which contains $A=\mathrm{L}^{\infty}(X)$ as a Cartan subalgebra, that is, $A \subset M$ is maximal abelian with expectation and the group of normalizing unitaries $\mathcal{N}_{M}(A)=\left\{u \in \mathcal{U}(M): u A u^{*}\right\}$ generates $M$ as a von Neumann algebra.

When $\Gamma \curvearrowright(X, \mu)$ is a nonsingular Borel action of a countable discrete group on a standard probability space, we will denote by $\mathcal{R}(\Gamma \curvearrowright X)$ the nonsingular orbit equivalence relation defined by

$$
\mathcal{R}(\Gamma \curvearrowright X)=\{(x, \gamma x) \mid \gamma \in \Gamma, x \in X\} .
$$

When the action $\Gamma \curvearrowright(X, \mu)$ is moreover essentially free, there is a canonical isomorphism of pairs of von Neumann algebras

$$
\left(\mathrm{L}^{\infty}(X) \subset \mathrm{L}(\mathcal{R}(\Gamma \curvearrowright X))\right) \cong\left(\mathrm{L}^{\infty}(X) \subset \mathrm{L}^{\infty}(X) \rtimes \Gamma\right) .
$$

For more information on nonsingular equivalence relations and their von Neumann algebras, we refer the reader to [FM75].

Strongly ergodic actions and full factors. We first recall the concept of strong ergodicity for group actions and equivalence relations.

Definition 2.4. Let $(X, \mu)$ be any standard probability space.

(i) Let $\Gamma$ be any countable discrete group and $\Gamma \curvearrowright(X, \mu)$ any ergodic nonsingular action. The action $\Gamma \curvearrowright(X, \mu)$ is said to be strongly ergodic if for any sequence $\left(C_{n}\right)_{n}$ of measurable subsets of $X$ such that $\lim _{n} \mu\left(\gamma C_{n} \triangle C_{n}\right)=0$ for all $\gamma \in \Gamma$, we have $\lim _{n} \mu\left(C_{n}\right)\left(1-\mu\left(C_{n}\right)\right)=0$.

(ii) Let $\mathcal{R}$ be any ergodic nonsingular equivalence relation defined on $(X, \mu)$. The equivalence relation $\mathcal{R}$ is said to be strongly ergodic if for any sequence $\left(C_{n}\right)_{n}$ of measurable subsets of $X$ such that $\lim _{n} \mu\left(g C_{n} \triangle C_{n}\right)=0$ for all $g \in[\mathcal{R}]$, we have $\lim _{n} \mu\left(C_{n}\right)(1-$ $\left.\mu\left(C_{n}\right)\right)=0$.

Put $A=\mathrm{L}^{\infty}(X)$ and fix any nonprincipal ultrafilter $\omega \in \beta(\mathbf{N}) \backslash \mathbf{N}$. Then the ergodic nonsingular action $\Gamma \curvearrowright(X, \mu)$ is strongly ergodic if and only if the ultraproduct action $\Gamma \curvearrowright A^{\omega}$ defined by $\gamma \cdot\left(a_{n}\right)^{\omega}=\left(\gamma \cdot a_{n}\right)^{\omega}$ is ergodic, that is, $\left(A^{\omega}\right)^{\Gamma}=\mathbf{C} 1$. Likewise, the ergodic nonsingular equivalence relation $\mathcal{R}$ defined on $(X, \mu)$ is strongly ergodic if and only if $\mathrm{L}(\mathcal{R})^{\prime} \cap A^{\omega}=\mathbf{C} 1$. We also have that the nonsingular action $\Gamma \curvearrowright(X, \mu)$ is strongly ergodic if and only if the nonsingular orbit equivalence relation $\mathcal{R}(\Gamma \curvearrowright X)$ is strongly ergodic.

Following Co74, we say that a factor $M$ with separable predual is full if $M_{\omega}=\mathrm{C} 1$ for some (or any) nonprincipal ultrafilter $\omega \in \beta(\mathbf{N}) \backslash \mathbf{N}$. By [AH12, Theorem 5.2], $M$ is full if and only if $M^{\prime} \cap M^{\omega}=\mathbf{C} 1$ for some (or any) nonprincipal ultrafilter $\omega \in \beta(\mathbf{N}) \backslash \mathbf{N}$. Observe that for any ergodic nonsingular equivalence relation $\mathcal{R}$ defined on a standard probability space $(X, \mu)$, if $\mathrm{L}(\mathcal{R})$ is full then $\mathcal{R}$ is strongly ergodic. 
Connes proved in [Co74, Theorem 2.12] that factors of type $\mathrm{III}_{0}$ are never full. Ueda showed in [Ue00, Corollary 11] that ergodic nonsingular equivalence relations of type $\mathrm{III}_{0}$ are never strongly ergodic. We give a short proof of Ueda's result. We refer to [Co72] for the type classification of factors.

Proposition 2.5 ([Ue00, Corollary 11]). Let $\mathcal{R}$ be any ergodic nonsingular equivalence relation defined on a standard probability space $(X, \mu)$. If $\mathcal{R}$ is of type $\mathrm{III}_{0}$, then $\mathcal{R}$ is not strongly ergodic.

Proof. Put $A=\mathrm{L}^{\infty}(X)$ and $M=\mathrm{L}(\mathcal{R})$. Assume that $\mathcal{R}$ is of type $\mathrm{III}_{0}$. Then $M$ is of type $\mathrm{III}_{0}$. Fix a nonprincipal ultrafilter $\omega$ on $\mathbf{N}$. Then $\mathcal{Z}\left(M^{\omega}\right) \neq \mathbf{C} 1$ by [AH12, Theorem 6.18]. Since $A^{\omega}$ is maximal abelian in $M^{\omega}$ by [Po95, Theorem A.1.2], we have

$$
\mathbf{C} 1 \neq \mathcal{Z}\left(M^{\omega}\right)=\left(M^{\omega}\right)^{\prime} \cap M^{\omega}=\left(M^{\omega}\right)^{\prime} \cap A^{\omega} \subset M^{\prime} \cap A^{\omega} .
$$

Since $M^{\prime} \cap A^{\omega} \neq \mathbf{C} 1, \mathcal{R}$ is not strongly ergodic.

Popa's intertwining-by-bimodules. In this subsection, we briefly recall Popa's intertwiningby-bimodules Po01, Po03. In the present paper, we will need a generalization of Popa's intertwining-by-bimodules to the framework of type III von Neumann algebras developed by the authors in [HI15. We will use the following terminology (see [HI15, Definition 4.1]).

Definition 2.6. Let $M$ be any $\sigma$-finite von Neumann algebra, $1_{A}$ and $1_{B}$ any nonzero projections in $M, A \subset 1_{A} M 1_{A}$ and $B \subset 1_{B} M 1_{B}$ any von Neumann subalgebras with faithful normal conditional expectations $\mathrm{E}_{A}: 1_{A} M 1_{A} \rightarrow A$ and $\mathrm{E}_{B}: 1_{B} M 1_{B} \rightarrow B$ respectively.

We say that $A$ embeds with expectation into $B$ inside $M$ and write $A \preceq_{M} B$ if there exist projections $e \in A$ and $f \in B$, a nonzero partial isometry $v \in e M f$ and a unital normal *homomorphism $\theta: e A e \rightarrow f B f$ such that the inclusion $\theta(e A e) \subset f B f$ is with expectation and $a v=v \theta(a)$ for all $a \in e A e$.

The main characterization of intertwining subalgebras we will use in this paper is the following result proven in [HI15, Theorem 4.3].

Theorem 2.7. Keep the same notation as in Definition [2.6 and moreover assume that $A$ is finite. Then the following conditions are equivalent.

(1) $A \preceq_{M} B$.

(2) There exists no net $\left(w_{i}\right)_{i \in I}$ of unitaries in $\mathcal{U}(A)$ such that $\mathrm{E}_{B}\left(b^{*} w_{i} a\right) \rightarrow 0$ in the $\sigma$ strong topology for all $a, b \in 1_{A} M 1_{B}$.

\section{Bi-exactness and Ozawa's condition (AO)}

Bi-exactness for discrete groups. Recall from Oz03 that a von Neumann algebra $M \subset$ $\mathbf{B}(H)$ satisfies condition (AO) if there exist unital $\sigma$-weakly dense $\mathrm{C}^{*}$-subalgebras $A \subset M$ and $B \subset M^{\prime}$ such that $A$ is locally reflexive and the map

$$
\nu: A \otimes_{\text {alg }} B \longrightarrow \mathbf{B}(H) / \mathbf{K}(H): a \otimes b \mapsto a b+\mathbf{K}(H)
$$

is continuous with respect to the minimal tensor norm. Recall that $A$ is locally reflexive or equivalently has property $C^{\prime \prime}$ (see e.g. [BO08, Section 9]) if for any $\mathrm{C}^{*}$-algebra $C$, the inclusion map $A^{* *} \otimes_{\text {alg }} C \hookrightarrow\left(A \otimes_{\min } C\right)^{* *}$ is continuous with respect to the minimal tensor norm. In this case, any $*$-homomorphism $\pi: A \otimes_{\min } C \rightarrow \mathbf{B}(K)$ has an extension $\widetilde{\pi}: A^{* *} \otimes_{\min } C \rightarrow \mathbf{B}(K)$ which is normal on $A^{* *} \otimes \mathbf{C} 1$ (since $\pi$ always has a canonical extension on $\left.\left(A \otimes_{\min } C\right)^{* *}\right)$.

We next recall the notion of bi-exactness for discrete groups which was introduced by Ozawa in Oz04 (using the terminology class $\mathcal{S}$ ) and intensively studied in [B008, Chapter 15]. Our definition is different from the original one, but it is equivalent to it and it is moreover adapted to the framework of discrete quantum groups [Is13, Definition 3.2]. 
Definition 3.1 ([BO08, Proposition 15.2.3(2)]). Let $\Gamma$ be any discrete group. We say that $\Gamma$ is bi-exact if there exists a $(\Gamma \times \Gamma)$-globally invariant unital $\mathrm{C}^{*}$-subalgebra $\mathcal{B} \subset \ell^{\infty}(\Gamma)$ such that the following two conditions are satisfied:

(i) The algebra $\mathcal{B}$ contains $c_{0}(\Gamma)$ so that the quotient $\mathcal{B}_{\infty}:=\mathcal{B} / c_{0}(\Gamma)$ is well-defined.

(ii) The left translation action $\Gamma \curvearrowright \ell^{\infty}(\Gamma)$ induces an amenable action $\Gamma \curvearrowright \mathcal{B}_{\infty}$ and the right translation action $\ell^{\infty}(\Gamma) \curvearrowleft \Gamma$ induces the trivial action on $\mathcal{B}_{\infty}$.

The class of bi-exact discrete groups includes amenable groups, free groups AO74, discrete subgroups of simple connected Lie groups of real rank one [Sk88] and Gromov word-hyperbolic groups $\mathrm{Oz03}$. Observe that for any bi-exact discrete group $\Gamma$, the group von Neumann algebra $\mathrm{L}(\Gamma)$ satisfies condition $(\mathrm{AO})$. We refer the reader to [BO08, Chapter 15] for more information on bi-exact discrete groups.

Ozawa's condition (AO) in crossed product von Neumann algebras. In this subsection, we prove a relative version of Ozawa's condition (AO) in the framework of crossed product von Neumann algebras. This result will be used in the proof of Theorem A

Let $\Gamma$ be any discrete group, $B \subset \mathcal{B}$ any inclusion of $\sigma$-finite von Neumann algebras and $\Gamma \curvearrowright \mathcal{B}$ any action that leaves globally invariant the subalgebra $B$. Denote by $M:=B \rtimes \Gamma$ and $\mathcal{M}=\mathcal{B} \rtimes \Gamma$ the corresponding crossed product von Neumann algebras, by $\mathrm{E}_{\mathcal{B}}: \mathcal{M} \rightarrow$ $\mathcal{B}$ the canonical faithful normal conditional expectation and by $e_{\mathcal{B}}: \mathrm{L}^{2}(\mathcal{M}) \rightarrow \mathrm{L}^{2}(\mathcal{B})$ the corresponding Jones projection. We use the notation and terminology of Section 2 for the standard forms $\left(\mathcal{B}, \mathrm{L}^{2}(\mathcal{B}), J^{\mathcal{B}}, \mathfrak{P}^{\mathcal{B}}\right)$ of $\mathcal{B}$ and $\left(\mathcal{M}, \mathrm{L}^{2}(\mathcal{M}), J^{\mathcal{M}}, \mathfrak{P}^{\mathcal{M}}\right)$ of $\mathcal{M}=\mathcal{B} \rtimes \Gamma$.

We define a nondegenerate (and possibly nonunital) $\mathrm{C}^{*}$-algebra and its multiplier $\mathrm{C}^{*}$-algebra inside $\mathbf{B}\left(\mathrm{L}^{2}(\mathcal{M})\right)$ by

$$
\begin{aligned}
\mathcal{K}_{\mathcal{B}} & :=\mathrm{C}^{*}\left\{a J^{\mathcal{M}} x J^{\mathcal{M}} e_{\mathcal{B}} b J^{\mathcal{M}} y J^{\mathcal{M}} \mid a, b, x, y \in B \rtimes_{\text {red }} \Gamma\right\} \subset \mathbf{B}\left(\mathrm{L}^{2}(\mathcal{M})\right) \\
\mathfrak{M}\left(\mathcal{K}_{\mathcal{B}}\right) & :=\left\{T \in \mathbf{B}\left(\mathrm{L}^{2}(\mathcal{M})\right) \mid T \mathcal{K}_{\mathcal{B}} \subset \mathcal{K}_{\mathcal{B}} \text { and } \mathcal{K}_{\mathcal{B}} T \subset \mathcal{K}_{\mathcal{B}}\right\} .
\end{aligned}
$$

where $\mathrm{C}^{*}\{\mathcal{Y}\} \subset \mathbf{B}\left(\mathrm{L}^{2}(\mathcal{M})\right)$ denotes the $\mathrm{C}^{*}$-subalgebra of $\mathbf{B}\left(\mathrm{L}^{2}(\mathcal{M})\right)$ generated by the subset $\mathcal{Y} \subset \mathbf{B}\left(\mathrm{L}^{2}(\mathcal{M})\right)$. We record the following elementary lemma.

Lemma 3.2. We have

$$
\mathcal{K}_{\mathcal{B}} \subset \mathbf{B}\left(\mathrm{L}^{2}(\mathcal{B})\right) \otimes_{\min } \mathbf{K}\left(\ell^{2}(\Gamma)\right) .
$$

Proof. Denote by $\sigma: \Gamma \curvearrowright \mathcal{B}$ the action and by $u: \Gamma \rightarrow \mathcal{U}\left(\mathrm{L}^{2}(\mathcal{B})\right)$ the canonical unitary representation implementing the action $\sigma$. Recall that $\mathrm{L}^{2}(\mathcal{M})=\mathrm{L}^{2}(\mathcal{B}) \otimes \ell^{2}(\Gamma)$. Regard $\mathcal{M}=$ $\mathcal{B} \rtimes \Gamma$ as generated by $\pi_{\sigma}(b)=\sum_{h \in \Gamma} \sigma_{h^{-1}}(b) \otimes P_{\mathbf{C} \delta_{h}}$ for $b \in \mathcal{B}$ and $1 \otimes \lambda_{g}$ for $g \in \Gamma$ where $P_{\mathbf{C} \delta_{h}}: \ell^{2}(\Gamma) \rightarrow \mathbf{C} \delta_{h}$ is the orthogonal projection onto $\mathbf{C} \delta_{h}$. We have $J^{\mathcal{M}}\left(1 \otimes \lambda_{g}\right) J^{\mathcal{M}}=u_{g} \otimes \rho_{g}$ for all $g \in \Gamma$. Let $\mathcal{C} \subset \mathbf{B}\left(\mathrm{L}^{2}(\mathcal{B})\right)$ be the $\mathrm{C}^{*}$-algebra generated by $B, J^{\mathcal{B}} B J^{\mathcal{B}}$ and $u_{g}$ for all $g \in \Gamma$. We will show that $\mathcal{K}=\mathcal{C} \otimes_{\min } \mathbf{K}\left(\ell^{2}(\Gamma)\right)$.

Recall that $e_{\mathcal{B}}=1 \otimes P_{\mathbf{C} \delta_{e}}$. For all $g, h \in \Gamma$, denote by $e_{g, h}: \mathbf{C} \delta_{h} \rightarrow \mathbf{C} \delta_{g}$ the partial isometry sending $\delta_{h}$ onto $\delta_{g}$. For all $a, b \in B$ and all $g, h, s, t \in \Gamma$, we have

$$
\begin{aligned}
\pi_{\sigma}(a)\left(J^{\mathcal{B}} b J^{\mathcal{B}} \otimes 1\right) e_{\mathcal{B}} & =e_{\mathcal{B}} \pi_{\sigma}(a)\left(J^{\mathcal{B}} b J^{\mathcal{B}} \otimes 1\right)=a J^{\mathcal{B}} b J^{\mathcal{B}} \otimes P_{\mathbf{C} \delta_{e}} \\
\left(1 \otimes \lambda_{g}\right)\left(u_{s} \otimes \rho_{s}\right) e_{\mathcal{B}}\left(1 \otimes \lambda_{h}\right)\left(u_{t} \otimes \rho_{t}\right) & =u_{s t} \otimes \lambda_{g} \rho_{s} P_{\mathbf{C} \delta_{e}} \lambda_{h} \rho_{t}=u_{s t} \otimes e_{g s^{-1}, h^{-1} t} .
\end{aligned}
$$

We then have

$$
\begin{aligned}
\mathcal{K}_{\mathcal{B}} & =\mathrm{C}^{*}\left\{a J^{\mathcal{M}} x J^{\mathcal{M}} e_{\mathcal{B}} b J^{\mathcal{M}} y J^{\mathcal{M}} \mid a, b, x, y \in B \rtimes_{\text {red }} \Gamma\right\} \\
& =\mathrm{C}^{*}\left\{a J^{\mathcal{B}} b J^{\mathcal{B}} u_{g} \otimes e_{s, t} \mid a, b \in B, g, s, t \in \Gamma\right\} \\
& =\mathcal{C} \otimes_{\min } \mathbf{K}\left(\ell^{2}(\Gamma)\right) .
\end{aligned}
$$

This finishes the proof of Lemma 3.2 
Consider now the following unital $*$-homomorphism:

$$
\nu_{\mathcal{B}}:\left(B \rtimes_{\text {red }} \Gamma\right) \otimes_{\text {alg }} J^{\mathcal{M}}\left(B \rtimes_{\text {red }} \Gamma\right) J^{\mathcal{M}} \rightarrow \mathfrak{M}\left(\mathcal{K}_{\mathcal{B}}\right) / \mathcal{K}_{\mathcal{B}}: a \otimes J^{\mathcal{M}} b J^{\mathcal{M}} \mapsto a J^{\mathcal{M}} b J^{\mathcal{M}}+\mathcal{K}_{\mathcal{B}}
$$

Ozawa proved in Oz04, Proposition 4.2] that when $\Gamma$ is bi-exact and $B=\mathcal{B}$ is finite and amenable, the map $\nu_{\mathcal{B}}$ is continuous with respect to the minimal tensor norm. This is nothing but a relative version of the condition $(\mathrm{AO})$ in the framework of crossed product von Neumann algebras. Observe that when $B=\mathcal{B}=\mathbf{C} 1$, continuity of $\nu_{\mathcal{B}}$ with respect to the minimal tensor norm implies that $\mathrm{L}(\Gamma)$ satisfies condition $(\mathrm{AO})$. Since $\mathcal{K}_{\mathcal{B}}$ is the smallest $\mathrm{C}^{*}$-algebra containing $1 \otimes c_{0}(\Gamma)$ and such that its multiplier algebra contains $B \rtimes_{\text {red }} \Gamma$ and $J^{\mathcal{M}}\left(B \rtimes_{\text {red }} \Gamma\right) J^{\mathcal{M}}$, we can easily generalize [z04, Proposition 4.2] as follows.

Proposition 3.3. Keep the same setting as above and assume that $\Gamma$ is bi-exact and $B$ is amenable. Then the map

$$
\nu_{\mathcal{B}}:\left(B \rtimes_{\text {red }} \Gamma\right) \otimes_{\text {alg }} J^{\mathcal{M}}\left(B \rtimes_{\text {red }} \Gamma\right) J^{\mathcal{M}} \rightarrow \mathfrak{M}\left(\mathcal{K}_{\mathcal{B}}\right) / \mathcal{K}_{\mathcal{B}}: a \otimes J^{\mathcal{M}} b J^{\mathcal{M}} \mapsto a J^{\mathcal{M}} b J^{\mathcal{M}}+\mathcal{K}_{\mathcal{B}}
$$

is well-defined and continuous with respect to the minimal tensor norm.

Proof. As in the proof of Lemma 3.2, regard $\mathcal{M}=\mathcal{B} \rtimes \Gamma$ as generated by $\pi_{\sigma}(\mathcal{B})$ and $(1 \otimes \lambda)(\Gamma)$. Since $B$ is amenable (i.e. semidiscrete), the map

$$
\pi_{\sigma}(B) \otimes_{\mathrm{alg}} J^{\mathcal{M}} \pi_{\sigma}(B) J^{\mathcal{M}} \rightarrow \mathbf{B}\left(\mathrm{L}^{2}(\mathcal{M})\right): \pi_{\sigma}(a) \otimes J^{\mathcal{M}} \pi_{\sigma}(b) J^{\mathcal{M}} \mapsto \pi_{\sigma}(a) J^{\mathcal{M}} \pi_{\sigma}(b) J^{\mathcal{M}}
$$

is continuous with respect to the minimal tensor norm. Then the proof of Oz04, Proposition 4.2] applies mutatis mutandis to show that the map $\nu_{\mathcal{B}}$ is continuous with respect to the minimal tensor norm.

We will apply Proposition 3.3 in Theorem 4.1 (in the case when $\mathcal{B}=B$ ) and in Theorem 4.2 (in the general case).

Ozawa's condition (AO) in ultraproduct von Neumann algebras. In this subsection, we prove a version of Ozawa's condition (AO) in the framework of ultraproduct von Neumann algebras. Although we will not use this result in this paper, we nevertheless mention it since we believe it is interesting in its own right.

We keep the same notation as in the previous subsection and we moreover assume that $B=\mathcal{B}$. Let $\omega \in \beta(\mathbf{N}) \backslash \mathbf{N}$ be any nonprincipal ultrafilter. Denote by $\left(M^{\omega}, \mathrm{L}^{2}\left(M^{\omega}\right), J^{M^{\omega}}, \mathfrak{P}^{M^{\omega}}\right)$ a standard form for $M^{\omega}$ and by $e_{B^{\omega}}: \mathrm{L}^{2}\left(M^{\omega}\right) \rightarrow \mathrm{L}^{2}\left(B^{\omega}\right)$ the Jones projection corresponding to the inclusion $B^{\omega} \subset M^{\omega}$. We define a (possibly degenerate and nonunital) $\mathrm{C}^{*}$-subalgebra $\mathcal{K}_{\omega}$ and its multiplier algebra $\mathfrak{M}\left(\mathcal{K}_{\omega}\right)$ inside $\mathbf{B}\left(\mathrm{L}^{2}\left(M^{\omega}\right)\right)$ by

$$
\begin{aligned}
\mathcal{K}_{\omega} & :=\mathrm{C}^{*}\left\{a J^{M^{\omega}} x J^{M^{\omega}} e_{B} b J^{M^{\omega}} y J^{M^{\omega}} \mid a, b, x, y \in B \rtimes_{\text {red }} \Gamma\right\} \subset \mathbf{B}\left(\mathrm{L}^{2}\left(M^{\omega}\right)\right) \\
\mathfrak{M}\left(\mathcal{K}_{\omega}\right) & :=\left\{T \in \mathbf{B}\left(\mathrm{L}^{2}\left(M^{\omega}\right)\right) \mid T \mathcal{K}_{\omega} \subset \mathcal{K}_{\omega} \text { and } \mathcal{K}_{\omega} T \subset \mathcal{K}_{\omega}\right\} .
\end{aligned}
$$

Recall from Proposition 3.3 (in the case when $B=\mathcal{B}$ with $\mathcal{K}:=\mathcal{K}_{\mathcal{B}}$ which is exactly Oz04, Proposition 4.2]) that the map

$$
\nu:\left(B \rtimes_{\text {red }} \Gamma\right) \otimes_{\text {alg }} J^{M}\left(B \rtimes_{\text {red }} \Gamma\right) J^{M} \rightarrow \mathfrak{M}(\mathcal{K}) / \mathcal{K}: a \otimes J^{M} b J^{M} \mapsto a J^{M} b J^{M}+\mathcal{K}
$$

is continuous with respect to the minimal tensor norm. We now state a version of Ozawa's condition $(\mathrm{AO})$ in the ultraproduct representation $\mathrm{L}^{2}\left(M^{\omega}\right)$.

Proposition 3.4. Keep the same setting as above and assume that $\Gamma$ is bi-exact and $B$ is amenable. Then the map

$$
\nu_{\omega}:\left(B \rtimes_{\text {red }} \Gamma\right) \otimes_{\text {alg }} J^{M^{\omega}}\left(B \rtimes_{\text {red }} \Gamma\right) J^{M^{\omega}} \rightarrow \mathfrak{M}\left(\mathcal{K}_{\omega}\right) / \mathcal{K}_{\omega}: a \otimes J^{M^{\omega}} b J^{M^{\omega}} \mapsto a J^{M^{\omega}} b J^{M^{\omega}}+\mathcal{K}_{\omega}
$$

is well-defined and continuous with respect to the minimal tensor norm. 
Proof. Put

$$
\begin{aligned}
C & :=\mathrm{C}^{*}\left\{B \rtimes_{\text {red }} \Gamma, J^{M}\left(B \rtimes_{\text {red }} \Gamma\right) J^{M}\right\} \subset \mathbf{B}\left(\mathrm{L}^{2}(M)\right) \\
C_{\omega} & :=\mathrm{C}^{*}\left\{B \rtimes_{\text {red }} \Gamma, J^{M^{\omega}}\left(B \rtimes_{\text {red }} \Gamma\right) J^{M^{\omega}}\right\} \subset \mathbf{B}\left(\mathrm{L}^{2}\left(M^{\omega}\right)\right) .
\end{aligned}
$$

Observe that $C+\mathcal{K}$ (resp. $\left.C_{\omega}+\mathcal{K}_{\omega}\right)$ is a $\mathrm{C}^{*}$-algebra since it is the sum of a $\mathrm{C}^{*}$-subalgebra and an ideal in $\mathfrak{M}(\mathcal{K})$ (resp. $\left.\mathfrak{M}\left(\mathcal{K}_{\omega}\right)\right)$.

Claim. There is a $*$-homomorphism $\theta: C+\mathcal{K} \rightarrow \mathbf{B}\left(\mathrm{L}^{2}\left(M^{\omega}\right)\right)$ such that $\theta(x)=x$ and $\theta\left(J^{M} y J^{M}\right)=J^{M^{\omega}} y J^{M^{\omega}}$ for all $x, y \in B \rtimes_{\text {red }} \Gamma$ and $\theta\left(e_{B}\right)=e_{B^{\omega}}$.

Proof of the Claim. Indeed, fix any faithful state $\varphi \in M_{*}$ such that $\varphi \circ \mathrm{E}_{B}=\varphi$ and denote by $p$ the support projection in $N=\prod^{\omega} M$ of the ultraproduct state $\varphi_{\omega} \in N_{*}$. By Lemma 2.2, $\pi^{\omega}\left(\left(e_{B}\right)_{n}\right)$ commutes with $p$ and $J^{N}$ and hence $\pi^{\omega}\left(\left(e_{B}\right)_{n}\right)$ commutes with $\widetilde{p}:=p J^{N} p J^{N}$. Since $\widetilde{p}$ commutes with $\pi^{\omega}(M)$ and $\pi^{\omega}\left(J^{M} M J^{M}\right)=J^{N} \pi^{\omega}(M) J^{N}, \widetilde{p}$ commutes with $\pi^{\omega}(C+\mathcal{K})$. Recall that $\widetilde{p} N \widetilde{p} \cong p N p \cong M^{\omega}$ and $\widetilde{p} \mathrm{~L}^{2}(M)_{\omega}=\mathrm{L}^{2}\left(M^{\omega}\right)$. Then the $*$-homomorphism

$$
\theta: C+\mathcal{K} \rightarrow \mathbf{B}\left(\mathrm{L}^{2}\left(M^{\omega}\right)\right): T \mapsto \widetilde{p} \pi^{\omega}(T) \widetilde{p}
$$

satisfies all the conditions of the Claim.

Since $\theta(C)=C_{\omega}$ and $\theta(\mathcal{K})=\mathcal{K}_{\omega}, \theta$ induces a $*$-homomorphism

$$
\widetilde{\theta}:(C+\mathcal{K}) / \mathcal{K} \rightarrow\left(C_{\omega}+\mathcal{K}_{\omega}\right) / \mathcal{K}_{\omega} \subset \mathfrak{M}\left(\mathcal{K}_{\omega}\right) / \mathcal{K}_{\omega} .
$$

Denote by $\iota:\left(B \rtimes_{\text {red }} \Gamma\right) \otimes_{\min } J^{M^{\omega}}\left(B \rtimes_{\text {red }} \Gamma\right) J^{M^{\omega}} \rightarrow\left(B \rtimes_{\text {red }} \Gamma\right) \otimes_{\min } J^{M}\left(B \rtimes_{\text {red }} \Gamma\right) J^{M}$ the tautological $*$-isomorphism. Then the composition map

$$
\nu_{\omega}=\tilde{\theta} \circ \nu \circ \iota:\left(B \rtimes_{\text {red }} \Gamma\right) \otimes_{\text {alg }} J^{M^{\omega}}\left(B \rtimes_{\text {red }} \Gamma\right) J^{M^{\omega}} \rightarrow \mathfrak{M}\left(\mathcal{K}_{\omega}\right) / \mathcal{K}_{\omega}
$$

is continuous with respect to the minimal tensor norm.

Weak exactness for $\mathrm{C}^{*}$-algebras. To obtain structural results for von Neumann algebras $M$ satisfying Ozawa's (relative) condition (AO) Oz03, Oz04, it is usually necessary to impose local reflexivity or exactness of the given unital $\sigma$-weakly dense $\mathrm{C}^{*}$-algebra in $M$. Observe that in the setting of Proposition 3.3 , the reduced crossed product $\mathrm{C}^{*}$-algebra $B \rtimes_{\text {red }} \Gamma$ need not be locally reflexive since it contains the von Neumann algebra $B$. To avoid this difficulty, Ozawa assumed in Oz04, Theorem 4.6] that $\Gamma$ is exact and $B$ is abelian so that $B \rtimes_{\text {red }} \Gamma$ is exact and hence locally reflexive. In Is12, the second named author introduced a notion of weak exactness for $\mathrm{C}^{*}$-algebras and could settle this problem. Namely, he generalized Oz04, Theorem 4.6] under the assumptions that $\Gamma$ is exact and $B$ is amenable (not necessarily abelian). The main idea behind this generalization was to use some exactness (or equivalently property $C^{\prime}$ ) of the opposite algebra $\left(B \rtimes_{\text {red }} \Gamma\right)^{\mathrm{op}}$, instead of local reflexivity of $B \rtimes_{\text {red }} \Gamma$. In the present paper, to study more general cases, we will make use of this notion of weak exactness for $\mathrm{C}^{*}$-algebras.

Recall from [Is12, Theorem 3.1.3(1)(ii)] that for an inclusion of a unital $\mathrm{C}^{*}$-algebra $A \subset M$ in a von Neumann algebra $M$, we say that $A$ is weakly exact in $M$ if for any unital $\mathrm{C}^{*}$-algebra $C$, any $*$-homomorphism $\pi: A \otimes_{\min } C \rightarrow \mathbf{B}(K)$ which is $\sigma$-weakly continuous on $A \otimes \mathbf{C} 1$ has an extension $\tilde{\pi}: A \otimes_{\min } C^{* *} \rightarrow \mathbf{B}(K)$ which is normal on $\mathbf{C} 1 \otimes C^{* *}$. In the case when $A=M$, we simply say that $M$ is weakly exact. Here we recall the following fundamental fact.

Proposition 3.5 ([Is12, Proposition 4.1.7]). Let $\Gamma$ be any exact discrete group, $B$ any $\sigma$-finite amenable (and hence weakly exact) von Neumann algebra and $\Gamma \curvearrowright B$ any action. Then the reduced crossed product $\mathrm{C}^{*}$-algebra $B \rtimes_{\mathrm{red}} \Gamma$ is weakly exact in $B \rtimes \Gamma$. If moreover $\Gamma$ is countable and $B$ has separable predual, then $B \rtimes \Gamma$ is weakly exact. 
Using this property, we prove an important lemma, which is a variant of [Oz03, Lemma 5] (see also [BO08, Proposition 15.1.6] and [Is12, Lemma 5.1.1]). The proof is essentially the same as the one of [BO08, Proposition 15.1.6] but we nevertheless include it for the reader's convenience.

Lemma 3.6. Let $M \subset \mathcal{M}$ be any inclusion of $\sigma$-finite von Neumann algebras with expectation and $\left(\mathcal{M}, \mathrm{L}^{2}(\mathcal{M}), J^{\mathcal{M}}, \mathfrak{P}^{\mathcal{M}}\right)$ a standard form for $\mathcal{M}$. Let $C \subset M$ be any unital $\sigma$-weakly dense $\mathrm{C}^{*}$-subalgebra, $p \in \mathcal{M}$ any nonzero projection and $\varphi: M \rightarrow p \mathcal{M} p$ any normal ucp map. We will use the identification $p \mathbf{B}\left(\mathrm{L}^{2}(\mathcal{M})\right) p=\mathbf{B}\left(p \mathrm{~L}^{2}(\mathcal{M})\right)$.

Assume that the following two conditions hold:

- The map

$$
\Phi: C \otimes_{\mathrm{alg}} J^{\mathcal{M}} C J^{\mathcal{M}} \rightarrow \mathbf{B}\left(p \mathrm{~L}^{2}(\mathcal{M})\right): \sum_{i=1}^{n} x_{i} \otimes J^{\mathcal{M}} y_{i} J^{\mathcal{M}} \mapsto \sum_{i=1}^{n} \varphi\left(x_{i}\right) J^{\mathcal{M}} y_{i} J^{\mathcal{M}} p
$$

is continuous with respect to the minimal tensor norm.

- The $\mathrm{C}^{*}$-algebra $C$ is locally reflexive or $C$ is weakly exact in $M$.

Then the ucp map $\varphi: M \rightarrow p \mathcal{M} p$ has a ucp extension $\widetilde{\varphi}: \mathbf{B}\left(\mathrm{L}^{2}(\mathcal{M})\right) \rightarrow\left(J^{\mathcal{M}} C J^{\mathcal{M}} p\right)^{\prime} \cap$ $\mathbf{B}\left(p \mathrm{~L}^{2}(\mathcal{M})\right)$.

Proof. To simplify the notation, we will write $J=J^{\mathcal{M}}$. Observe that $\Phi=\nu \circ\left(\varphi \otimes \operatorname{id}_{J C J}\right)$ where $\nu: p \mathcal{M} p \otimes_{\text {alg }} J C J \rightarrow \mathbf{B}\left(p \mathrm{~L}^{2}(\mathcal{M})\right)$ is the multiplication map. We first prove the following result.

Claim. The ucp map $\Phi: C \otimes_{\min } J C J \rightarrow \mathbf{B}\left(p \mathrm{~L}^{2}(\mathcal{M})\right)$ can be extended to a ucp map $\widetilde{\Phi}$ : $M \otimes \min J C J \rightarrow \mathbf{B}\left(p \mathrm{~L}^{2}(\mathcal{M})\right)$ which is normal $M \otimes \mathbf{C} 1$. In particular, we have $\widetilde{\Phi}(x \otimes 1)=\varphi(x)$ for all $x \in M$.

Proof of the Claim. Indeed, let $(\pi, V, K)$ be a minimal Stinespring dilation for $\Phi: C \otimes_{\text {min }}$ $J C J \rightarrow \mathbf{B}\left(p \mathrm{~L}^{2}(\mathcal{M})\right)$, that is, $\pi: C \otimes_{\min } J C J \rightarrow \mathbf{B}(K)$ is a unital *-representation and $V: p \mathrm{~L}^{2}(\mathcal{M}) \rightarrow K$ is an isometry such that the subspace $\pi\left(C \otimes_{\min } J C J\right) V p \mathrm{~L}^{2}(\mathcal{M})$ is dense in $K$ and $\Phi(x)=V^{*} \pi(x) V$ for all $x \in C$. By minimality of $(\pi, V, K)$ and since $\Phi$ is $\sigma$-weakly continuous on $C \otimes \mathbf{C} 1$ (resp. $\mathbf{C} 1 \otimes J C J$ ), we have that $\pi$ is also $\sigma$-weakly continuous on $C \otimes \mathbf{C} 1$ $($ resp. $\mathbf{C} 1 \otimes J C J)$. Indeed, it suffices to notice that for all $c_{1}, c_{2} \in C$, all $x, y \in C \otimes_{\min } J C J$ and all $\xi, \eta \in p \mathrm{~L}^{2}(\mathcal{M})$, we have

$$
\begin{aligned}
\left\langle\pi\left(c_{1} \otimes J c_{2} J\right) \pi(x) V \xi, \pi(y) V \eta\right\rangle_{K} & =\left\langle V^{*} \pi\left(y^{*}\left(c_{1} \otimes J c_{2} J\right) x\right) V \xi, \eta\right\rangle_{K} \\
& =\left\langle\Phi\left(y^{*}\left(c_{1} \otimes J c_{2} J\right) x\right) \xi, \eta\right\rangle_{K} .
\end{aligned}
$$

Since $C$ is assumed to be locally reflexive or weakly exact in $M$ (which is equivalent to saying that $J C J$ is weakly exact in $J M J)$, the unital $*$-homomorphism $\pi: C \otimes_{\min } J C J \rightarrow \mathbf{B}(K)$ always has an extension $\tilde{\pi}: C^{* *} \otimes_{\min } J C J \rightarrow \mathbf{B}(K)$ which is normal on $C^{* *} \otimes \mathbf{C}$ 1. Observe that we do not need $\sigma$-weak continuity on $\mathbf{C} 1 \otimes J C J$ when $C$ is locally reflexive.

Let $z \in C^{* *}$ be the central projection such that $z C^{* *}=M$ canonically and let $z_{i} \in C$ be a bounded net converging to $z$ in the $\sigma$-weak topology in $C^{* *}$. Observe that $z_{i} \rightarrow 1_{M} \sigma$-weakly in $M$ and recall that $\tilde{\pi}$ (resp. $\pi$ ) is $\sigma$-weakly continuous on $C^{* *} \otimes \mathbf{C} 1$ (resp. $C \otimes \mathbf{C} 1 \subset M \otimes \mathbf{C} 1$ ). We then have that

$$
\tilde{\pi}(z \otimes 1)=\lim _{i} \pi\left(z_{i} \otimes 1\right)=\pi\left(1_{M} \otimes 1\right)=1
$$

and hence $\widetilde{\pi}((1-z) \otimes 1)=0$. Since $\widetilde{\pi}$ is a $*$-homomorphism, it satisfies $\widetilde{\pi}((z \otimes 1) x)=\widetilde{\pi}(x)$ for all $x \in C^{* *} \otimes_{\min } J C J$. In particular, we have $\left.\tilde{\pi}((z \otimes 1) \cdot)\right|_{C \otimes_{\min } J C J}=\pi$. Using moreover the identification $M \otimes_{\min } J C J=z C^{* *} \otimes_{\min } J C J$, we obtain that the unital $*$-homomorphism $\tilde{\pi}((z \otimes 1) \cdot): M \otimes_{\min } J C J \rightarrow \mathbf{B}(K)$ is an extension of $\pi: C \otimes_{\min } J C J \rightarrow \mathbf{B}(K)$ which is normal 
on $M \otimes \mathbf{C} 1$. Therefore the ucp map $\widetilde{\Phi}=\operatorname{Ad}\left(V^{*}\right) \circ \widetilde{\pi}((z \otimes 1) \cdot): M \otimes_{\min } J C J \rightarrow \mathbf{B}\left(p \mathrm{~L}^{2}(\mathcal{M})\right)$ is an extension of $\Phi: C \otimes_{\min } J C J \rightarrow \mathbf{B}\left(p \mathrm{~L}^{2}(\mathcal{M})\right)$ which is normal on $M \otimes \mathbf{C} 1$. In particular, we have $\widetilde{\Phi}(x \otimes 1)=\varphi(x)$ for all $x \in M$.

We next apply Arveson's extension theorem to the ucp map $\widetilde{\Phi}: M \otimes_{\min } J C J \rightarrow \mathbf{B}\left(p \mathrm{~L}^{2}(\mathcal{M})\right)$ and we obtain a ucp extension map that we still denote by $\widetilde{\Phi}: \mathbf{B}\left(\mathrm{L}^{2}(\mathcal{M})\right) \otimes_{\min } J C J \rightarrow$ $\mathbf{B}\left(p \mathrm{~L}^{2}(\mathcal{M})\right)$. Since $\left.\widetilde{\Phi}\right|_{\mathbf{C} 1 \otimes J C J}: \mathbf{C} 1 \otimes J C J \rightarrow \mathbf{B}\left(p \mathrm{~L}^{2}(\mathcal{M})\right): 1 \otimes J x J \mapsto J x J p$ is a unital *-homomorphism, $\mathbf{C} 1 \otimes J C J$ is contained in the multiplicative domain of $\widetilde{\Phi}$ (see e.g. [BO08, Section 1.5]). Therefore, for all $u \in \mathcal{U}(C)$ and all $x \in \mathbf{B}\left(\mathrm{L}^{2}(\mathcal{M})\right)$, we have $\Phi(x \otimes 1) J u J p=\Phi(x \otimes 1) \Phi(1 \otimes J u J)=\Phi(x \otimes J u J)=\Phi(1 \otimes J u J) \Phi(x \otimes 1)=J u J p \Phi(x \otimes 1)$ and hence $\Phi\left(\mathbf{B}\left(\mathrm{L}^{2}(\mathcal{M})\right) \otimes 1\right) \subset(J C J p)^{\prime} \cap \mathbf{B}\left(p \mathrm{~L}^{2}(\mathcal{M})\right)$. Thus, $\widetilde{\varphi}:=\Phi(\cdot \otimes 1): \mathbf{B}\left(\mathrm{L}^{2}(\mathcal{M})\right) \rightarrow$ $(J C J p)^{\prime} \cap \mathbf{B}\left(p \mathrm{~L}^{2}(\mathcal{M})\right)$ is the desired ucp extension map.

\section{Proofs of Theorem A and Corollary B}

We first prove two intermediate results, namely Theorems 4.1 and 4.2 , from which we will deduce Theorem $\mathrm{A}$. While these two results are independent from each other, their proofs are in fact very similar and use Ozawa's condition (AO) for crossed product von Neumann algebras from Section 3 ,

Theorem 4.1 below is a spectral gap rigidity result for subalgebras with expectation $N \subset M$ of crossed product von Neumann algebras $M=B \rtimes \Gamma$ arising from arbitrary actions of bi-exact discrete groups on amenable von Neumann algebras.

Theorem 4.1. Let $\Gamma$ be any bi-exact discrete group, $B$ any amenable $\sigma$-finite von Neumann algebra and $\Gamma \curvearrowright B$ any action. Put $M:=B \rtimes \Gamma$. Let $p \in M$ be any nonzero projection and $N \subset p M p$ any von Neumann subalgebra with expectation. Let $\omega \in \beta(\mathbf{N}) \backslash \mathbf{N}$ be any nonprincipal ultrafilter.

Then at least one of the following conditions holds true.

- The von Neumann algebra $N$ has a nonzero amenable direct summand.

- We have $N^{\prime} \cap p M^{\omega} p \subset p\left(B^{\omega} \rtimes \Gamma\right) p$.

Proof. Assume that $N^{\prime} \cap p M^{\omega} p \not \subset p\left(B^{\omega} \rtimes \Gamma\right) p$. Let $Y \in N^{\prime} \cap p M^{\omega} p$ be such that $Y \notin p\left(B^{\omega} \rtimes \Gamma\right) p$. Up to replacing $Y$ by $Y-\mathrm{E}_{B^{\omega} \rtimes \Gamma}(Y) \neq 0$ which still lies in $N^{\prime} \cap p M^{\omega} p$, we may assume that $Y \in$ $N^{\prime} \cap p M^{\omega} p, Y \neq 0$ and $\mathrm{E}_{B^{\omega} \rtimes \Gamma}(Y)=0$. Put $y=\mathrm{E}_{\omega}\left(Y^{*} Y\right) \in\left(N^{\prime} \cap p M p\right)^{+}$. Define the nonzero spectral projection $p_{0}:=\mathbf{1}_{\left[\frac{1}{2}\|y\|_{\infty},\|y\|_{\infty}\right]}(y) \in N^{\prime} \cap p M p$ and put $c:=\left(y p_{0}\right)^{-1 / 2} \in N^{\prime} \cap p M p$. We have

$$
\mathrm{E}_{\omega}\left((Y c)^{*}(Y c)\right)=\mathrm{E}_{\omega}\left(c Y^{*} Y c\right)=c \mathrm{E}_{\omega}\left(Y^{*} Y\right) c=c y c=p_{0} .
$$

Up to replacing $Y$ by $Y c$ which still lies in $N^{\prime} \cap p M^{\omega} p$, we may assume that $Y \in N^{\prime} \cap p M^{\omega} p$, $Y \neq 0, \mathrm{E}_{B^{\omega} \rtimes \Gamma}(Y)=0$ and $\mathrm{E}_{\omega}\left(Y^{*} Y\right)=p_{0}$. Write $Y=\left(y_{n}\right)^{\omega}$ for some $\left(y_{n}\right)_{n} \in \mathcal{M}^{\omega}(M)$. Observe that $\sigma$-weak $\lim _{n \rightarrow \omega} y_{n}^{*} y_{n}=\mathrm{E}_{\omega}\left(Y^{*} Y\right)=p_{0}$.

Denote by $\left(M, \mathrm{~L}^{2}(M), J^{M}, \mathfrak{P}^{M}\right)$ a standard form for $M=B \rtimes \Gamma$ as in Section 2. To further simplify the notation, we will write $J=J^{M}$ and $\mathfrak{P}=\mathfrak{P}^{M}$. Define the cp map

$$
\Psi: \mathbf{B}\left(\mathrm{L}^{2}(M)\right) \rightarrow \mathbf{B}\left(\mathrm{L}^{2}(M)\right): T \mapsto \sigma \text {-weak } \lim _{n \rightarrow \omega} y_{n}^{*} T y_{n}
$$

Observe that $\Psi(1)=p_{0}$. Since $\Psi$ is a cp map and $\Psi(1)=p_{0}$ is a projection, we have $\Psi(T)=$ $\Psi(1) \Psi(T) \Psi(1)=p_{0} \Psi(T) p_{0}$ for every $T \in \mathbf{B}\left(\mathrm{L}^{2}(M)\right)$ and hence $\Psi\left(\mathbf{B}\left(\mathrm{L}^{2}(M)\right)\right) \subset \mathbf{B}\left(p_{0} \mathrm{~L}^{2}(M)\right)$ using the identification $p_{0} \mathbf{B}\left(\mathrm{L}^{2}(M)\right) p_{0}=\mathbf{B}\left(p_{0} \mathrm{~L}^{2}(M)\right)$. We will then regard $\Psi: \mathbf{B}\left(\mathrm{L}^{2}(M)\right) \rightarrow$ $\mathbf{B}\left(p_{0} \mathrm{~L}^{2}(M)\right)$ as a ucp map. Observe that $\Psi(x)=\mathrm{E}_{\omega}\left(Y^{*} x Y\right)$ for all $x \in M$ and hence $\Psi(M) \subset$ $p_{0} M p_{0}$ and $\left.\Psi\right|_{M}$ is normal. Moreover, observe that $\left.\Psi\right|_{N}: N \rightarrow \mathbf{B}\left(p_{0} \mathrm{~L}^{2}(M)\right): x \mapsto x p_{0}$ and 
$\left.\Psi\right|_{J M J}: J M J \rightarrow \mathbf{B}\left(p_{0} \mathrm{~L}^{2}(M)\right): J x J \mapsto J x J p_{0}$ are unital $*$-homomorphisms. We will denote by $\psi:=\left.\Psi\right|_{M}: M \rightarrow p_{0} M p_{0}: x \mapsto \Psi(x)$.

Let $\mathcal{K}_{B}$ as in Proposition 3.3 (for $\mathcal{B}=B$ ). For all $a, b \in M$, we have

$$
\mathrm{E}_{B^{\omega}}\left(b^{*} Y a\right)=\mathrm{E}_{B^{\omega}}\left(\mathrm{E}_{B^{\omega} \rtimes \Gamma}\left(b^{*} Y a\right)\right)=\mathrm{E}_{B^{\omega}}\left(b^{*} \mathrm{E}_{B^{\omega} \rtimes \Gamma}(Y) a\right)=0 .
$$

Since $0=\mathrm{E}_{B^{\omega}}\left(b^{*} Y a\right)=\left(\mathrm{E}_{B}\left(b^{*} y_{n} a\right)\right)^{\omega}$, we obtain that $\mathrm{E}_{B}\left(b^{*} y_{n} a\right) \rightarrow 0 \sigma$-strongly as $n \rightarrow \omega$. Choose any cyclic unit vector $\xi \in \mathfrak{P}$ such that $e_{B} \xi=\xi$. For all $a, b, c, d \in M$, we have

$$
\begin{aligned}
\left|\left\langle\Psi\left(a e_{B} b\right) c \xi, d \xi\right\rangle_{\mathrm{L}^{2}(M)}\right| & =\lim _{n \rightarrow \omega}\left|\left\langle y_{n}^{*} a e_{B} b y_{n} c \xi, d \xi\right\rangle_{\mathrm{L}^{2}(M)}\right| \\
& =\lim _{n \rightarrow \omega}\left|\left\langle e_{B} b y_{n} c \xi, e_{B} a^{*} y_{n} d \xi\right\rangle_{\mathrm{L}^{2}(M)}\right| \\
& =\lim _{n \rightarrow \omega}\left|\left\langle e_{B} b y_{n} c e_{B} \xi, e_{B} a^{*} y_{n} d e_{B} \xi\right\rangle_{\mathrm{L}^{2}(M)}\right| \\
& =\lim _{n \rightarrow \omega}\left|\left\langle\mathrm{E}_{B}\left(b y_{n} c\right) e_{B} \xi, \mathrm{E}_{B}\left(a^{*} y_{n} d\right) e_{B} \xi\right\rangle_{\mathrm{L}^{2}(M)}\right| \\
& =\lim _{n \rightarrow \omega}\left|\left\langle\mathrm{E}_{B}\left(b y_{n} c\right) \xi, \mathrm{E}_{B}\left(a^{*} y_{n} d\right) \xi\right\rangle_{\mathrm{L}^{2}(M)}\right| \\
& \leq \lim _{n \rightarrow \omega}\left\|\mathrm{E}_{B}\left(b y_{n} c\right) \xi\right\|_{\mathrm{L}^{2}(M)}\left\|\mathrm{E}_{B}\left(a^{*} y_{n} d\right) \xi\right\|_{\mathrm{L}^{2}(M)} \\
& =0 .
\end{aligned}
$$

This implies that $\Psi\left(a e_{B} b\right)=0$. By construction, we have $M=B \rtimes \Gamma$ and hence $e_{B}$ corresponds to the projection $1 \otimes P_{\mathbf{C} \delta_{e}}$. Taking $a=\lambda_{g}$ and $b=\lambda_{h}$ for $g, h \in \Gamma$, we then obtain $\Psi\left(\mathbf{C} 1 \otimes \mathbf{K}\left(\ell^{2}(\Gamma)\right)\right)=0$. Since $\Psi$ is a ucp map, we obtain $\Psi\left(\mathbf{B}\left(\mathrm{L}^{2}(B)\right) \otimes_{\min } \mathbf{K}\left(\ell^{2}(\Gamma)\right)\right)=0$ and hence $\Psi\left(\mathcal{K}_{B}\right)=0$ using Lemma 3.2, Define the ucp map $\bar{\Psi}: \mathfrak{M}\left(\mathcal{K}_{B}\right) / \mathcal{K}_{B} \rightarrow \mathbf{B}\left(p_{0} \mathrm{~L}^{2}(M)\right)$ : $a+\mathcal{K}_{B} \mapsto \Psi(a)$.

Using Proposition 3.3 in the case when $B=\mathcal{B}$, we may then define the ucp composition map

$$
\Phi=\bar{\Psi} \circ \nu:\left(B \rtimes_{\text {red }} \Gamma\right) \otimes_{\min } J\left(B \rtimes_{\text {red }} \Gamma\right) J \rightarrow \mathbf{B}\left(p_{0} \mathrm{~L}^{2}(M)\right): a \otimes J b J \mapsto \Psi(a J b J) .
$$

Since $\left.\Psi\right|_{J M J}$ is a unital $*$-homomorphism and since $\psi=\left.\Psi\right|_{M}$ by definition, we have $\Phi(a \otimes$ $J b J)=\Psi(a J b J)=\Psi(a) \Psi(J b J)=\psi(a) J b J p_{0}$ for all $a, b \in B \rtimes_{\text {red }} \Gamma$. Since

$$
\left(J\left(B \rtimes_{\text {red }} \Gamma\right) J p_{0}\right)^{\prime} \cap \mathbf{B}\left(p_{0} \mathrm{~L}^{2}(M)\right)=p_{0}(J M J)^{\prime} p_{0} \cap \mathbf{B}\left(p_{0} \mathrm{~L}^{2}(M)\right)=p_{0} M p_{0},
$$

Proposition 3.5 and Lemma 3.6 imply that the normal ucp map $\psi: M \rightarrow p_{0} M p_{0}$ has a ucp extension $\widetilde{\psi}: \mathbf{B}\left(\mathrm{L}^{2}(M)\right) \rightarrow p_{0} M p_{0}$. Observe that $N p_{0} \subset p_{0} M p_{0}$ is still with expectation by [HU15, Proposition 2.2]. Denote by $\mathrm{E}_{N p_{0}}: p_{0} M p_{0} \rightarrow N p_{0}$ a faithful normal conditional expectation. Define the unital $*$-homomorphism $\iota: N \rightarrow N p_{0}: x \mapsto x p_{0}$ and denote by $z \in \mathcal{Z}(N)$ the unique central projection such that $\operatorname{ker}(\iota)=N z^{\perp}$. Then $N z \cong N p_{0}$ and $N z p_{0}=N p_{0}$. Define the ucp map $\Theta=\iota^{-1} \circ \mathrm{E}_{N p_{0}} \circ \widetilde{\psi}(z \cdot z): \mathbf{B}\left(z \mathrm{~L}^{2}(M)\right) \rightarrow N z$. Since $\left.\Theta\right|_{N z}=\operatorname{id}_{N z}, \Theta$ is a norm one projection and hence $N z$ is amenable. We have therefore proved that if $N^{\prime} \cap p M^{\omega} p \not \subset p\left(B^{\omega} \rtimes \Gamma\right) p$, then $N$ has a nonzero amenable direct summand.

Theorem 4.2. Let $\Gamma$ be any bi-exact discrete group, $B \subset \mathcal{B}$ any inclusion of $\sigma$-finite von Neumann algebras with expectation and $\Gamma \curvearrowright \mathcal{B}$ any action that leaves the subalgebra $B$ globally invariant. Assume moreover that $B$ is amenable. Put $M:=B \rtimes \Gamma \subset \mathcal{B} \rtimes \Gamma=: \mathcal{M}$. Let $p \in M$ be any nonzero projection and $N \subset p M p$ any von Neumann subalgebra with expectation.

Then at least one of the following conditions holds true.

- The von Neumann algebra $N$ is amenable.

- We have $A \preceq_{\mathcal{M}} \mathcal{B}$ for any finite von Neumann subalgebra $A \subset N^{\prime} \cap p \mathcal{M} p$ with expectation. 
Proof. Since the proof is very similar to the one of Theorem 4.1, we will simply sketch it and point out the necessary changes compared to Theorem 4.1. Denote by $\left(\mathcal{M}, \mathrm{L}^{2}(\mathcal{M}), J^{\mathcal{M}}, \mathfrak{P}^{\mathcal{M}}\right)$ a standard form for $\mathcal{M}=\mathcal{B} \rtimes \Gamma$ as in Section 2. Suppose that there exists a finite von Neumann subalgebra $A \subset N^{\prime} \cap p \mathcal{M} p$ with expectation such that $A \npreceq \mathcal{M} \mathcal{B}$. Observe that since $N^{\prime} \cap p \mathcal{M} p \subset p \mathcal{M} p$ is with expectation, so is $A \subset p \mathcal{M} p$. We will show $N$ is amenable. We will use the identifications $p \mathbf{B}\left(\mathrm{L}^{2}(M)\right) p=\mathbf{B}\left(p \mathrm{~L}^{2}(M)\right)$ and $p \mathbf{B}\left(\mathrm{L}^{2}(\mathcal{M})\right) p=\mathbf{B}\left(p \mathrm{~L}^{2}(\mathcal{M})\right)$.

Take a net of unitaries $\left(u_{i}\right)_{i \in I}$ in $\mathcal{U}(A)$ as in Theorem 2.7(ii) such that $\mathrm{E}_{\mathcal{B}}\left(b^{*} u_{i} a\right) \rightarrow 0 \sigma$-strongly for any $a, b \in \mathcal{M}$. Fix a cofinal ultrafilter $\mathcal{U}$ on the directed set $I$ and define the ucp map

$$
\Psi: \mathbf{B}\left(\mathrm{L}^{2}(\mathcal{M})\right) \rightarrow \mathbf{B}\left(p \mathrm{~L}^{2}(\mathcal{M})\right): T \mapsto \sigma \text {-weak } \lim _{i \rightarrow \mathcal{U}} u_{i}^{*} T u_{i} .
$$

Observe that $\left.\Psi\right|_{\mathcal{M}}: \mathcal{M} \rightarrow p \mathcal{M} p$ is normal. Indeed, since $A \subset p \mathcal{M} p$ is finite and with expectation and since $\mathcal{M}$ is $\sigma$-finite, there exists a faithful state $\varphi \in(p \mathcal{M} p)_{*}$ such that $A \subset(p \mathcal{M} p)^{\varphi}$. Since $u_{i} \in \mathcal{U}(A)$ for all $i \in I$, this implies that $(\varphi \circ \Psi)(p x p)=\varphi(x)$ for all $x \in p \mathcal{M} p$. Since $\varphi$ is faithful and normal and since $\Psi=\Psi(p \cdot p)$, it follows that $\left.\Psi\right|_{\mathcal{M}}: \mathcal{M} \rightarrow p \mathcal{M} p$ is indeed normal. Moreover, we have $\Psi(x)=x$ for all $x \in N$.

Let $\mathcal{K}_{\mathcal{B}}$ be as in Proposition 3.3. By a reasoning entirely similar to the one of the proof of Theorem 4.1, we have $\Psi\left(\mathcal{K}_{\mathcal{B}}\right)=0$. Define the ucp map $\bar{\Psi}: \mathfrak{M}\left(\mathcal{K}_{\mathcal{B}}\right) / \mathcal{K}_{\mathcal{B}} \rightarrow \mathbf{B}\left(p \mathrm{~L}^{2}(\mathcal{M})\right)$ : $a+\mathcal{K}_{\mathcal{B}} \mapsto \Psi(a)$. Using Proposition 3.3, we may then define the ucp composition map

$$
\bar{\Psi} \circ \nu_{\mathcal{B}}:\left(B \rtimes_{\text {red }} \Gamma\right) \otimes_{\min } J^{\mathcal{M}}\left(B \rtimes_{\text {red }} \Gamma\right) J^{\mathcal{M}} \rightarrow \mathbf{B}\left(p \mathrm{~L}^{2}(\mathcal{M})\right): a \otimes J^{\mathcal{M}} b J^{\mathcal{M}} \mapsto \Psi\left(a J^{\mathcal{M}} b J^{\mathcal{M}}\right) .
$$

Proposition 3.5 and Lemma 3.6 imply that the normal ucp map $\psi:=\left.\Psi\right|_{M}: M \rightarrow p \mathcal{M} p$ has a ucp extension $\widetilde{\psi}: \mathbf{B}\left(\mathrm{L}^{2}(\mathcal{M})\right) \rightarrow\left(J^{\mathcal{M}}\left(B \rtimes_{\text {red }} \Gamma\right) J^{\mathcal{M}} p\right)^{\prime} \cap \mathbf{B}\left(p \mathrm{~L}^{2}(\mathcal{M})\right)$. Denote by $e_{M}$ : $\mathrm{L}^{2}(\mathcal{M}) \rightarrow \mathrm{L}^{2}(M)$ the Jones projection corresponding to the inclusion $M \subset \mathcal{M}$. We then have the identifications $e_{M} \mathbf{B}\left(p \mathrm{~L}^{2}(\mathcal{M})\right) e_{M}=\mathbf{B}\left(p \mathrm{~L}^{2}(M)\right)$ and $e_{M} J^{\mathcal{M}} e_{M}=J^{M}$. Then we have

$$
e_{M}\left(\left(J^{\mathcal{M}}\left(B \rtimes_{\text {red }} \Gamma\right) J^{\mathcal{M}} p\right)^{\prime} \cap \mathbf{B}\left(p \mathrm{~L}^{2}(\mathcal{M})\right)\right) e_{M}=\left(J^{M}\left(B \rtimes_{\text {red }} \Gamma\right) J^{M} p\right)^{\prime} \cap \mathbf{B}\left(p \mathrm{~L}^{2}(M)\right)=p M p
$$

and hence the ucp map $\widetilde{\Psi}:=e_{M} \widetilde{\psi}(\cdot) e_{M}: \mathbf{B}\left(\mathrm{L}^{2}(\mathcal{M})\right) \rightarrow p M p$ takes indeed values in $p M p$. Moreover, we have $\widetilde{\Psi}(x)=x$ for all $x \in N$. If we denote by $\mathrm{E}_{N}: p M p \rightarrow N$ a faithful normal conditional expectation, the ucp map $\Theta=\mathrm{E}_{N} \circ \widetilde{\Psi}(p \cdot p): \mathbf{B}\left(p \mathrm{~L}^{2}(\mathcal{M})\right) \rightarrow N$ is a norm one projection. Therefore, $N$ is amenable.

Proof of Theorem $A$. Suppose that $N$ has no amenable direct summand. Then by Theorem 4.1. we have $N^{\prime} \cap p M^{\omega} p \subset p\left(B^{\omega} \rtimes \Gamma\right) p$. We then apply Theorem 4.2 to $N$ in the case when $\mathcal{B}:=B^{\omega}$ and we obtain $A \preceq_{B^{\omega} \rtimes \Gamma} B^{\omega}$ for any finite von Neumann subalgebra $A \subset N^{\prime} \cap p M^{\omega} p$ with expectation.

Proof of Corollary $B$. Let $N \subset M$ be any von Neumann subalgebra with expectation such that $N^{\prime} \cap M^{\omega}$ has no type I direct summand. Denote by $p \in \mathcal{Z}(N)$ the unique central projection such that $N p$ has no amenable direct summand and $N(1-p)$ is amenable. Assume by contradiction that $p \neq 0$. Then $(N p)^{\prime} \cap p M^{\omega} p \subset p\left(B^{\omega} \rtimes \Gamma\right) p$ by Theorem $\AA$ and $(N p)^{\prime} \cap p M^{\omega} p=p\left(N^{\prime} \cap\right.$ $\left.M^{\omega}\right) p$ has no type I direct summand. By [CS78, Corollary 8] (see also [HS90, Theorem 11.1]), $(N p)^{\prime} \cap p M^{\omega} p$ contains a copy of the hyperfinite $\mathrm{II}_{1}$ factor $R$ with expectation. We then have $R \preceq_{B^{\omega} \rtimes \Gamma} B^{\omega}$ by Theorem $\mathrm{A}$. Since $R$ is of type $\mathrm{II}_{1}$ and $B^{\omega}$ is abelian and hence of type I, we obtain a contradiction. Therefore, $p=0$ and $N$ is amenable.

\section{Proof of Theorem C}

We start by proving a useful lemma which can be regarded as a generalization of the first part of the proof of [Ho15, Proposition C]. 
Lemma 5.1. Let $\mathcal{R}$ be any strongly ergodic nonsingular equivalence relation defined on a standard probability space $(X, \mu)$. Put $A=\mathrm{L}^{\infty}(X)$ and $M=\mathrm{L}(\mathcal{R})$. Denote by $\mathrm{E}_{A}: M \rightarrow A$ the unique faithful normal conditional expectation. Fix any faithful state $\tau \in A_{*}$ and put $\varphi=\tau \circ \mathrm{E}_{A} \in M_{*}$.

If $M$ is not full, then there exists a sequence of unitaries $u_{n} \in \mathcal{U}(M)$ such that the following conditions hold:

(i) $\lim _{n}\left\|u_{n} \varphi-\varphi u_{n}\right\|=0$,

(ii) $\lim _{n}\left\|x u_{n}-u_{n} x\right\|_{\varphi}=0$ for all $x \in M$ and

(iii) $\lim _{n}\left\|\mathrm{E}_{A}\left(x u_{n} y\right)\right\|_{\varphi}=0$ for all $x, y \in M$.

Proof. Assume that $M$ is not full. Then $M^{\prime} \cap\left(M^{\omega}\right)^{\varphi^{\omega}}$ is diffuse by [HR14, Corollary 2.6] for any nonprincipal ultrafilter $\omega \in \beta(\mathbf{N}) \backslash \mathbf{N}$. Then a combination of the first part of the proof of [HR14, Theorem A] and Lemma 2.1 shows that there exists a sequence of unitaries $u_{n} \in \mathcal{U}(M)$ such that the following conditions hold:

(i) $\lim _{n}\left\|u_{n} \varphi-\varphi u_{n}\right\|=0$,

(ii) $\lim _{n}\left\|x u_{n}-u_{n} x\right\|_{\varphi}=0$ for all $x \in M$ and

(iii) $u_{n} \rightarrow 0 \sigma$-weakly as $n \rightarrow \infty$.

It remains to prove that Conditions (i), (ii), (iii) imply that $\lim _{n}\left\|\mathrm{E}_{A}\left(x u_{n} y\right)\right\|_{\varphi}=0$ for all $x, y \in M$. The rest of the proof is entirely analogous to the first part of the proof of [Ho15, Proposition $\mathrm{C}$ ] and we only give the details for the sake of completeness. Observe that for every nonprincipal ultrafilter $\omega \in \beta(\mathbf{N}) \backslash \mathbf{N}$, Condition (i) implies that $\left(u_{n}\right)_{n} \in \mathcal{M}^{\omega}(M)$ and Conditions (ii) and (iii) imply that $\left(u_{n}\right)^{\omega} \in M^{\prime} \cap\left(M^{\omega}\right)^{\varphi^{\omega}}$ and $\varphi^{\omega}\left(\left(u_{n}\right)^{\omega}\right)=0$. We start by proving the following claim.

Claim. We have $\lim _{n}\left\|\mathrm{E}_{A}\left(u_{n}\right)\right\|_{\varphi}=0$

Proof of the Claim. Let $g \in[\mathcal{R}]$ be any element and denote by $u_{g} \in \mathcal{U}(\mathrm{L}(\mathcal{R}))$ the corresponding unitary element. Since $u_{g} \mathrm{E}_{A}\left(u_{n}\right) u_{g}^{*}=\mathrm{E}_{A}\left(u_{g} u_{n} u_{g}^{*}\right)$, we have

$$
\begin{aligned}
\left\|\mathrm{E}_{A}\left(u_{n}\right) u_{g}^{*}-u_{g}^{*} \mathrm{E}_{A}\left(u_{n}\right)\right\|_{\varphi} & =\left\|u_{g} \mathrm{E}_{A}\left(u_{n}\right) u_{g}^{*}-\mathrm{E}_{A}\left(u_{n}\right)\right\|_{\varphi} \\
& =\left\|\mathrm{E}_{A}\left(u_{g} u_{n} u_{g}^{*}-u_{n}\right)\right\|_{\varphi} \\
& \leq\left\|u_{g} u_{n} u_{g}^{*}-u_{n}\right\|_{\varphi} \\
& =\left\|u_{n} u_{g}^{*}-u_{g}^{*} u_{n}\right\|_{\varphi} \rightarrow 0 \text { as } n \rightarrow \infty .
\end{aligned}
$$

Define $\mathcal{E}=\operatorname{span}\left\{a u_{g} \mid a \in A, g \in[\mathcal{R}]\right\}$ and observe that $\mathcal{E}$ is a unital $\sigma$-strongly dense *subalgebra of $M$. The above calculation implies that $\lim _{n}\left\|x \mathrm{E}_{A}\left(u_{n}\right)-\mathrm{E}_{A}\left(u_{n}\right) x\right\|_{\varphi}=0$ for every $x \in \mathcal{E}$. Let $\omega \in \beta(\mathbf{N}) \backslash \mathbf{N}$ be any nonprincipal ultrafilter. For every $x \in \mathcal{E}$, we have $\left\|x \mathrm{E}_{A^{\omega}}\left(\left(u_{n}\right)^{\omega}\right)-\mathrm{E}_{A^{\omega}}\left(\left(u_{n}\right)^{\omega}\right) x\right\|_{\varphi^{\omega}}=\lim _{n \rightarrow \omega}\left\|x \mathrm{E}_{A}\left(u_{n}\right)-\mathrm{E}_{A}\left(u_{n}\right) x\right\|_{\varphi}=0$ and hence we have $x \mathrm{E}_{A^{\omega}}\left(\left(u_{n}\right)^{\omega}\right)=\mathrm{E}_{A^{\omega}}\left(\left(u_{n}\right)^{\omega}\right) x$. Since $\mathcal{E}$ is $\sigma$-strongly dense in $M$, this further implies that $x \mathrm{E}_{A^{\omega}}\left(\left(u_{n}\right)^{\omega}\right)=\mathrm{E}_{A^{\omega}}\left(\left(u_{n}\right)^{\omega}\right) x$ for every $x \in M$. Since $\mathcal{R}$ is strongly ergodic, this implies that $\mathrm{E}_{A^{\omega}}\left(\left(u_{n}\right)^{\omega}\right)=\varphi^{\omega}\left(\left(u_{n}\right)^{\omega}\right) 1=0$ and hence $\lim _{n \rightarrow \omega}\left\|\mathrm{E}_{A}\left(u_{n}\right)\right\|_{\varphi}=\left\|\mathrm{E}_{A^{\omega}}\left(\left(u_{n}\right)^{\omega}\right)\right\|_{\varphi^{\omega}}=0$. Since this is true for every $\omega \in \beta(\mathbf{N}) \backslash \mathbf{N}$, we finally obtain that $\lim _{n}\left\|\mathrm{E}_{A}\left(u_{n}\right)\right\|_{\varphi}=0$.

We can now finish the proof of Lemma 5.1. Let $g \in[\mathcal{R}]$ be any element such that $g^{2}=1$. Put $X_{g}=\{s \in X \mid g \cdot s=s\}$ and observe that $u_{g}=u_{g}^{*}, z_{g}:=\mathrm{E}_{A}\left(u_{g}\right)=\mathbf{1}_{X_{g}}$ and $u_{g}^{*} z_{g}=z_{g} u_{g}^{*}=z_{g}$. Since $A$ is abelian and hence tracial, a combination of the fact that $u_{g}^{*} z_{g} \in A$ and the Claim implies that

$$
\left\|\mathrm{E}_{A}\left(u_{n} u_{g}^{*}\right) z_{g}\right\|_{\varphi}=\left\|\mathrm{E}_{A}\left(u_{n} u_{g}^{*} z_{g}\right)\right\|_{\varphi}=\left\|\mathrm{E}_{A}\left(u_{n}\right) u_{g}^{*} z_{g}\right\|_{\varphi} \leq\left\|\mathrm{E}_{A}\left(u_{n}\right)\right\|_{\varphi} \rightarrow 0 \quad \text { as } \quad n \rightarrow \infty .
$$

Denote by $\mathcal{J}$ the nonempty directed set (for the inclusion) of all the families of projections $\left(z_{i}\right)_{i \in I}$ in $A$ such that $z_{i} \leq 1-z_{g}, z_{i} \perp z_{j}$ for all $i \neq j \in I$ and $z_{i} \perp u_{g} z_{j} u_{g}^{*}$ for all $i, j \in I$. 
By Zorn's lemma, let $\left(z_{i}\right)_{i \in I}$ be a maximal element in $\mathcal{J}$. Put $z=\sum_{i \in I} z_{i}$ and assume by contradiction that $z+u_{g} z u_{g}^{*} \neq 1-z_{g}$. Put $e=1-z_{g}-z-u_{g} z u_{g}^{*} \neq 0$. Since $g^{2}=1$, we have $u_{g} e u_{g}^{*}=e$. Since $e \leq 1-z_{g}=\mathbf{1}_{\{s \in X \mid g \cdot s \neq s\}}$, we can find $0 \neq z^{\prime} \leq e$ such that $z^{\prime} \perp u_{g} z^{\prime} u_{g}^{*}$. Then the family $\left(\left(z_{i}\right)_{i \in I}, z^{\prime}\right)$ is in $\mathcal{J}$ and this contradicts the maximality of the family $\left(z_{i}\right)_{i \in I}$ in $\mathcal{J}$. Therefore, we have $z+u_{g} z u_{g}^{*}=1-z_{g}$. A calculation entirely analogous to Ho15, Proposition C, Equation (6.6)] shows that

$$
\begin{aligned}
\left\|\mathrm{E}_{A}\left(u_{n} u_{g}^{*}\right)\left(1-z_{g}\right)\right\|_{\varphi}^{2} & =\left\|\mathrm{E}_{A}\left(u_{n} u_{g}^{*}\right)\left(z+u_{g} z u_{g}^{*}\right)\right\|_{\varphi}^{2} \\
& =\left\|\mathrm{E}_{A}\left(u_{n} u_{g}^{*}\right) z\right\|_{\varphi}^{2}+\left\|\mathrm{E}_{A}\left(u_{n} u_{g}^{*}\right) u_{g} z u_{g}^{*}\right\|_{\varphi}^{2} \\
& =\left\|\mathrm{E}_{A}\left(u_{n} u_{g}^{*}\right)\left(z-u_{g} z u_{g}^{*}\right)\right\|_{\varphi}^{2} \\
& =\left\|\mathrm{E}_{A}\left(\left(z u_{n}-u_{n} z\right) u_{g}^{*}\right)\right\|_{\varphi}^{2} .
\end{aligned}
$$

Since $z u_{n}-u_{n} z \rightarrow 0 \sigma$-strongly as $n \rightarrow \infty$, we also have that $\mathrm{E}_{A}\left(\left(z u_{n}-u_{n} z\right) u_{g}^{*}\right) \rightarrow 0 \sigma$-strongly as $n \rightarrow \infty$. The above calculation implies that $\lim _{n}\left\|\mathrm{E}_{A}\left(u_{n} u_{g}^{*}\right)\left(1-z_{g}\right)\right\|_{\varphi}=0$. This further implies that

$$
\limsup _{n}\left\|\mathrm{E}_{A}\left(u_{n} u_{g}^{*}\right)\right\|_{\varphi}^{2}=\underset{n}{\limsup }\left(\left\|\mathrm{E}_{A}\left(u_{n} u_{g}^{*}\right) z_{g}\right\|_{\varphi}^{2}+\left\|\mathrm{E}_{A}\left(u_{n} u_{g}^{*}\right)\left(1-z_{g}\right)\right\|_{\varphi}^{2}\right)=0 .
$$

Define $\mathcal{F}=\operatorname{span}\left\{a u_{g} \mid a \in A, g \in[\mathcal{R}], g^{2}=1\right\}$. By the proof of [FM75, Theorem 1], it follows that $\mathcal{F}$ is a $\sigma$-strongly dense linear $*$-subspace of $M$. The previous reasoning shows that $\lim _{n}\left\|\mathrm{E}_{A}\left(u_{n} x\right)\right\|_{\varphi}=0$ for every $x \in \mathcal{F}$. Let $\omega \in \beta(\mathbf{N}) \backslash \mathbf{N}$ be any nonprincipal ultrafilter. For every $x \in \mathcal{F}$, we have $\left\|\mathrm{E}_{A^{\omega}}\left(\left(u_{n}\right)^{\omega} x\right)\right\|_{\varphi^{\omega}}=\lim _{n \rightarrow \omega}\left\|\mathrm{E}_{A}\left(u_{n} x\right)\right\|_{\varphi}=0$ and hence $\mathrm{E}_{A^{\omega}}\left(\left(u_{n}\right)^{\omega} x\right)=0$. Since $\mathcal{F}$ is $\sigma$-strongly dense in $M$, this further implies that $\mathrm{E}_{A^{\omega}}\left(\left(u_{n}\right)^{\omega} x\right)=0$ for every $x \in M$. Using Condition (ii), we also have $\mathrm{E}_{A^{\omega}}\left(x\left(u_{n}\right)^{\omega} y\right)=\mathrm{E}_{A^{\omega}}\left(\left(u_{n}\right)^{\omega} x y\right)=0$ for every $x, y \in M$. This implies that $\lim _{n \rightarrow \omega}\left\|\mathrm{E}_{A}\left(x u_{n} y\right)\right\|_{\varphi}=\left\|\mathrm{E}_{A^{\omega}}\left(x\left(u_{n}\right)^{\omega} y\right)\right\|_{\varphi^{\omega}}=0$. Since this is true for every $\omega \in \beta(\mathbf{N}) \backslash \mathbf{N}$, we finally obtain that $\lim _{n}\left\|\mathrm{E}_{A}\left(x u_{n} y\right)\right\|_{\varphi}=0$ for all $x, y \in M$.

Proof of Theorem $\square$. Simply write $B=\mathrm{L}^{\infty}(X)$ and $M=B \rtimes \Gamma$. Assume by contradiction that $M$ is not full. Fix any nonprincipal ultrafilter $\omega \in \beta(\mathbf{N}) \backslash \mathbf{N}$. Since $\Gamma \curvearrowright(X, \mu)$ is strongly ergodic, Lemma 5.1 shows that there exists $u \in \mathcal{U}\left(M_{\omega}\right)$ such that $\mathrm{E}_{B^{\omega}}\left(u \lambda_{s}\right)=0$ for every $s \in \Gamma$. Then for every $s \in \Gamma$, we have

$$
\mathrm{E}_{B^{\omega}}\left(\mathrm{E}_{B^{\omega} \rtimes \Gamma}(u) \lambda_{s}\right)=\mathrm{E}_{B^{\omega}}\left(\mathrm{E}_{B^{\omega} \rtimes \Gamma}\left(u \lambda_{s}\right)\right)=\mathrm{E}_{B^{\omega}}\left(u \lambda_{s}\right)=0 .
$$

This implies that $\mathrm{E}_{B^{\omega} \rtimes \Gamma}(u)=0$. Since $M$ is a nonamenable factor, Theorem $\mathrm{A}$ shows that $M_{\omega} \subset M^{\prime} \cap M^{\omega} \subset B^{\omega} \rtimes \Gamma$ and hence $u \in \mathcal{U}\left(B^{\omega} \rtimes \Gamma\right)$. We then have $u=\mathrm{E}_{B^{\omega} \rtimes \Gamma}(u)=0$. This is a contradiction.

\section{Group measure space type III factors With no Central Sequence}

Definition 6.1. Let $G$ be any locally compact second countable group. Let $G \curvearrowright(X, \mu)$ and $G \curvearrowright(Y, \nu)$ be any nonsingular Borel actions on standard probability spaces. We say that

- $G \curvearrowright(Y, \nu)$ is a measurable quotient of $G \curvearrowright(X, \mu)$ if, after discarding null $G$-invariant Borel subsets, there exists a $G$-equivariant Borel quotient map $q: X \rightarrow Y$ such that $\left[q_{*} \mu\right]=[\nu]$.

- $G \curvearrowright(Y, \nu)$ is measurably conjugate to $G \curvearrowright(X, \mu)$ if, after discarding null $G$-invariant Borel subsets, there exists a $G$-equivariant Borel isomorphism $\theta: X \rightarrow Y$ such that $\left[\theta_{*} \mu\right]=[\nu]$.

Let $G$ be any locally compact second countable group and $H<G$ any closed subgroup. Endowed with the quotient topology, $G / H$ is a continuous $G$-space, that is, the action $G \curvearrowright G / H$ defined by $(g, h H) \mapsto g h H$ is continuous. The quotient space $G / H$ carries, up to equivalence, a unique $G$-quasi-invariant regular Borel probability measure $\nu \in \operatorname{Prob}(G / H)$. Any such 
$G$-quasi-invariant regular Borel probability measure is associated with a rho-function for the pair $(G, H)$ (see e.g. [BdlHV08, Appendix B]). The action $G \curvearrowright G / H$ is indeed a measurable quotient of the translation action $G \curvearrowright G$ (see [BdlHV08, Theorem B.1.4]).

Let $G$ be any noncompact connected simple Lie group and $P<G$ any minimal parabolic subgroup (e.g. $G=\mathrm{SL}_{n}(\mathbf{R})$ and $P=$ subgroup of upper triangular matrices, for $n \geq 2$ ). Fix a $G$-quasi-invariant Borel regular probability measure $\nu \in \operatorname{Prob}(G / P)$. Denote by $\Delta_{P}: P \rightarrow \mathbf{R}_{+}^{*}$ the modular homomorphism and observe that $\Delta_{P}(P)=\mathbf{R}_{+}^{*}$ (this follows from BdlHV08, Proposition B.1.6 (ii)] and [Zi84, Proposition 4.3.2]). Put $L=\operatorname{ker}\left(\Delta_{P}\right)$. The Radon-Nikodym cocycle associated with the action $G \curvearrowright G / P$ is the map defined by

$$
\Omega: G \times G / P \rightarrow \mathbf{R}:(g, h P) \mapsto \log \left(\frac{\mathrm{d} g_{*} \nu}{\mathrm{d} \nu}(h P)\right) .
$$

Observe that $\Omega: G \times G / P \rightarrow \mathbf{R}$ is a continuous map by [BdlHV08, Theorem B.1.4]. The Maharam extension $G \curvearrowright G / P \times \mathbf{R}$ is the continuous action defined by

$$
g \cdot(h P, t)=(g h P, t+\Omega(g, h P)) .
$$

By [BdlHV08, Lemma B.1.3], we have $\Omega(g, P)=\left(\log \circ \Delta_{P}\right)(g)$ for every $g \in P$. Since moreover $\left(\log \circ \Delta_{P}\right)(P)=\mathbf{R}$, the Maharam extension $G \curvearrowright G / P \times \mathbf{R}$ is transitive and the stabilizer of the point $(P, 0)$ is equal to $L$. The mapping

$$
\theta: G / L \rightarrow G / P \times \mathbf{R}: g L \mapsto(g P, \Omega(g, P))
$$

is a well-defined $G$-equivariant homeomorphism that yields a measurable conjugacy between the action $G \curvearrowright G / L$ and the Maharam extension $G \curvearrowright G / P \times \mathbf{R}$. Therefore, we have proved the following useful fact.

Proposition 6.2 (see [BN11, Proposition 4.7]). The Maharam extension of $G \curvearrowright G / P$ is measurably conjugate to $G \curvearrowright G / L$.

From now on, fix $n \geq 2, G=\mathrm{SL}_{n}(\mathbf{R})$ and $\Lambda=\mathrm{SL}_{n}(\mathbf{Q})$ and denote by $P<G$ the minimal subgroup of upper triangular matrices. By [BISG15, Theorem A and Proposition 7.4], the translation action $\Lambda \curvearrowright G$ is strongly ergodic and so is the nonsingular action $\Lambda \curvearrowright G / P$ (recall that strong ergodicity is stable under taking measurable quotients). Fix a surjective group homomorphism $\pi: \mathbf{F}_{\infty} \rightarrow \Lambda$ such that $\operatorname{ker}(\pi)<\mathbf{F}_{\infty}$ is a nonamenable subgroup. For simplicity, write $\Gamma=\mathbf{F}_{\infty}$.

Put $X=[0,1]^{\Gamma}$ and $\mu=\operatorname{Leb}^{\otimes \Gamma}$ and consider the Bernoulli shift action $\Gamma \curvearrowright X$ defined by $\gamma \cdot\left(x_{\gamma^{\prime}}\right)_{\gamma^{\prime} \in \Gamma}=\left(x_{\gamma^{-1} \gamma^{\prime}}\right)_{\gamma^{\prime} \in \Gamma}$. Observe that $\Gamma \curvearrowright X$ preserves the Borel probability measure $\mu$ and is essentially free and strongly ergodic. Since $\operatorname{ker}(\pi)$ is nonamenable, the restricted action $\operatorname{ker}(\pi) \curvearrowright X$ is also strongly ergodic. Since $\operatorname{ker}(\pi)<\mathbf{F}_{\infty}$ is a nonamenable free subgroup and hence not inner amenable, the crossed product $\mathrm{II}_{1}$ factor $\mathrm{L}^{\infty}(X) \rtimes \operatorname{ker}(\pi)$ is full by Ch81. Define the action $\Gamma \curvearrowright X \times G / P$ by

$$
\gamma \cdot(x, h P)=(\gamma x, \pi(\gamma) h P) .
$$

Observe that $\Gamma \curvearrowright X \times G / P$ quasi-preserves the product measure $\mu \otimes \nu$ and is essentially free.

Theorem 6.3. Keep the same notation as above. The following assertions hold true:

(i) The nonsingular action $\Gamma \curvearrowright X \times G / P$ is essentially free and strongly ergodic and its Maharam extension $\Gamma \curvearrowright X \times G / P \times \mathbf{R}$ is also essentially free and strongly ergodic.

(ii) The group measure space factor $M=\mathrm{L}^{\infty}(X \times G / P) \rtimes \Gamma$ is a full type $\mathrm{III}_{1}$ factor and its continuous core $\mathrm{c}(M)$ is a full type $\mathrm{II}_{\infty}$ factor.

Proof. (i) As we already pointed out, the nonsingular action $\Gamma \curvearrowright X \times G / P$ is essentially free and so is its Maharam extension $\Gamma \curvearrowright X \times G / P \times \mathbf{R}$. 
We next prove that the nonsingular action $\Gamma \curvearrowright X \times G$ defined by $\gamma \cdot(x, h)=(\gamma x, \pi(\gamma) h)$ is strongly ergodic. Put $A=\mathrm{L}^{\infty}(X)$ and $B=\mathrm{L}^{\infty}(G)$ so that $\mathrm{L}^{\infty}(X \times G)=A \bar{\otimes} B$. Write $N=(A \bar{\otimes} B) \rtimes \Gamma$. Fix a nonprincipal ultrafilter $\omega \in \beta(\mathbf{N}) \backslash \mathbf{N}$. We need to show that $N^{\prime} \cap(A \bar{\otimes} B)^{\omega}=\mathbf{C} 1$. Observe that $(A \bar{\otimes} B) \rtimes \operatorname{ker}(\pi)=(A \rtimes \operatorname{ker}(\pi)) \bar{\otimes} B$ and $N^{\prime} \cap(A \bar{\otimes} B)^{\omega} \subset$ $(A \rtimes \operatorname{ker}(\pi))^{\prime} \cap((A \rtimes \operatorname{ker}(\pi)) \bar{\otimes} B)^{\omega}$. Since $A \rtimes \operatorname{ker}(\pi)$ is a full type $\mathrm{II}_{1}$ factor, Co75, Theorem 2.1] implies that $(A \rtimes \operatorname{ker}(\pi))^{\prime} \cap((A \rtimes \operatorname{ker}(\pi)) \bar{\otimes} B)^{\omega}=B^{\omega}$ and hence $N^{\prime} \cap(A \bar{\otimes} B)^{\omega}=N^{\prime} \cap B^{\omega}=$ $\left(B^{\omega}\right)^{\Lambda}$. By [BISG15, Theorem A], the nonsingular action $\Lambda \curvearrowright G$ is strongly ergodic, that is, $\left(B^{\omega}\right)^{\Lambda}=\mathbf{C} 1$. This implies that $N^{\prime} \cap(A \bar{\otimes} B)^{\omega}=\mathbf{C} 1$ and hence the nonsingular action $\Gamma \curvearrowright X \times G$ is strongly ergodic.

Since the nonsingular action $\Gamma \curvearrowright X \times G / P$ is a quotient of the strongly ergodic nonsingular action $\Gamma \curvearrowright X \times G$, it follows that $\Gamma \curvearrowright X \times G / P$ is also strongly ergodic. Consider the Maharam extension $\Lambda \curvearrowright G / P \times \mathbf{R}$ of the nonsingular action $\Lambda \curvearrowright G / P$. By Proposition 6.2. the Maharam extension $\Lambda \curvearrowright G / P \times \mathbf{R}$ is measurably conjugate to the nonsingular action $\Lambda \curvearrowright G / L$ where $L=\operatorname{ker}\left(\Delta_{P}\right)$ and $\Delta_{P}: P \rightarrow \mathbf{R}_{+}^{*}$ is the modular homomorphism. Since $\Gamma \curvearrowright X$ is pmp, the action $\Gamma \curvearrowright X \times G / L$ defined by $\gamma \cdot(x, h L)=(\gamma x, \pi(\gamma) h L)$ can be identified with the Maharam extension of the nonsingular action $\Gamma \curvearrowright X \times G / P$. Since the nonsingular action $\Gamma \curvearrowright X \times G / L$ is a quotient of the strongly ergodic nonsingular action $\Gamma \curvearrowright X \times G$, it follows that $\Gamma \curvearrowright X \times G / L$ is also strongly ergodic. Therefore, the Maharam extension $\Gamma \curvearrowright X \times G / P \times \mathbf{R}$ of the nonsingular action $\Gamma \curvearrowright X \times G / P$ is strongly ergodic.

(ii) This is a consequence of Theorem C

Keep the same notation as above. Fix $0<\lambda<1$, put $T=\frac{2 \pi}{|\log \lambda|}$ and identify $\mathbf{T}=\mathbf{R} /(T \mathbf{Z})$. Define the nonsingular action $\Gamma \curvearrowright X \times G / P \times \mathbf{T}$ by

$$
\gamma \cdot(x, h P, t+T \mathbf{Z})=(\gamma x, \pi(\gamma) h P, t+\Omega(\pi(\gamma), h P)+T \mathbf{Z}) .
$$

Observe that the nonsingular action $\Gamma \curvearrowright X \times G / P \times \mathbf{T}$ is a measurable quotient of the nonsingular action $\Gamma \curvearrowright X \times G / P \times \mathbf{R}$ and hence is strongly ergodic by Theorem 6.3(i). Moreover, we have a canonical identification

$$
\mathrm{L}^{\infty}(X \times G / P \times \mathbf{T}) \rtimes \Gamma=M \rtimes_{\sigma_{T}^{\varphi}} \mathbf{Z} .
$$

It follows that $\mathrm{L}^{\infty}(X \times G / P \times \mathbf{T}) \rtimes \Gamma$ is a type $\mathrm{III}_{\lambda}$ factor by [Co85, Lemma 1]. Observe that $\mathrm{L}^{\infty}(X \times G / P \times \mathbf{T}) \rtimes \Gamma$ is full by Theorem C. Alternatively, since $\mathrm{c}(M)$ is full by Theorem 6.3(ii), $\mathrm{L}^{\infty}(X \times G / P \times \mathbf{T}) \rtimes \Gamma=M \rtimes_{\sigma_{T}^{\varphi}} \mathbf{Z}$ is full by [TU14, Lemma 6].

Proof of Corollary D. This is a consequence of Theorem 6.3 and the above construction.

\section{FURTHER REMARKS}

In [HR14, the first named author and Raum investigated the asymptotic structure of Shlyakhtenko's free Araki-Woods factors [Sh96]. Among other things, they proved in HR14, Theorem A] that any diffuse von Neumann algebra $M$ with separable predual satisfying Ozawa's condition (AO) is $\omega$-solid, that is, for any von Neumann subalgebra with expectation $N \subset M$ such that the relative commutant $N^{\prime} \cap M^{\omega}$ is diffuse, we have that $N$ is amenable. The proof was based on a combination of Ozawa's $\mathrm{C}^{*}$-algebraic techniques and an analysis of the relative commutant $N^{\prime} \cap M^{\omega}$ and its centralizer [HR14, Theorem 2.3] (see [Io12a, Lemma 2.7] for the tracial case).

In this subsection, we observe that $\omega$-solidity can be easily obtained using the same proof as the one of Theorem 4.1 without relying on the analysis of the relative commutant $N^{\prime} \cap M^{\omega}$ from [HR14, Theorem 2.3]. We moreover remove the separability assumption of the predual. 
Theorem 7.1 ([HR14, Theorem A]). Let $M$ be any diffuse $\sigma$-finite von Neumann algebra satisfying Ozawa's condition $(\mathrm{AO})$. Let $p \in M$ be any nonzero projection and $N \subset p M p$ any von Neumann subalgebra with expectation.

Then at least one of the following conditions holds true:

- The von Neumann algebra $N$ has a nonzero amenable direct summand.

- We have $N^{\prime} \cap p M^{\omega} p \subset p M p$. In that case, $N^{\prime} \cap p M^{\omega} p=N^{\prime} \cap p M p$ is moreover discrete.

Proof. Suppose that $N$ has no amenable direct summand. Then the exact same argument as in the proof of Theorem 4.1 using Ozawa's condition (AO) in lieu of Proposition 3.3 shows that $N^{\prime} \cap p M^{\omega} p \subset p M p$ and hence $N^{\prime} \cap p M^{\omega} p=N^{\prime} \cap p M p$. Since $M$ is diffuse and solid [Oz03, Theorem 6] (see also [VV05, Theorem 2.5]), it follows that $p M p$ is also diffuse and solid. Since $N \subset p M p$ has no amenable direct summand, it follows that $N^{\prime} \cap p M p$ is necessarily discrete.

In view of Proposition 3.4, we finally observe the following condition (AO) in the ultraproduct representation.

Proposition 7.2. Let $M$ be any $\sigma$-finite von Neumann algebra and $\omega \in \beta(\mathbf{N}) \backslash \mathbf{N}$ any nonprincipal ultrafilter. Denote by $\left(M, \mathrm{~L}^{2}(M), J^{M}, \mathfrak{P}^{M}\right)$ (resp. $\left.\left(M^{\omega}, \mathrm{L}^{2}\left(M^{\omega}\right), J^{M^{\omega}}, \mathfrak{P}^{M^{\omega}}\right)\right)$ a standard form for $M$ (resp. $\left.M^{\omega}\right)$. Assume there are unital $\mathrm{C}^{*}$-subalgebras $A, B \subset M$ such that the map

$$
\nu: A \otimes_{\mathrm{alg}} J^{M} B J^{M} \rightarrow \mathbf{B}\left(\mathrm{L}^{2}(M)\right) / \mathbf{K}\left(\mathrm{L}^{2}(M)\right): a \otimes J^{M} b J^{M} \mapsto a J^{M} b J^{M}+\mathbf{K}\left(\mathrm{L}^{2}(M)\right)
$$

is continuous with respect to the minimal tensor norm.

Then the map

$\nu_{\omega}: A \otimes_{\text {alg }} J^{M^{\omega}} B J^{M^{\omega}} \rightarrow \mathbf{B}\left(\mathrm{L}^{2}\left(M^{\omega}\right)\right) / \mathbf{K}\left(\mathrm{L}^{2}\left(M^{\omega}\right)\right): a \otimes J^{M^{\omega}} b J^{M^{\omega}} \mapsto a J^{M^{\omega}} b J^{M^{\omega}}+\mathbf{K}\left(\mathrm{L}^{2}\left(M^{\omega}\right)\right)$

is continuous with respect to the minimal tensor norm.

Proof. The proof is a variation of the one of Proposition 3.4. Put

$$
\begin{aligned}
C & :=\mathrm{C}^{*}\left\{M, J^{M} M J^{M}\right\} \subset \mathbf{B}\left(\mathrm{L}^{2}(M)\right) \\
C_{\omega} & :=\mathrm{C}^{*}\left\{M, J^{M^{\omega}} M J^{M^{\omega}}\right\} \subset \mathbf{B}\left(\mathrm{L}^{2}\left(M^{\omega}\right)\right) .
\end{aligned}
$$

Observe that $C+\mathbf{K}\left(\mathrm{L}^{2}(M)\right)$ (resp. $C_{\omega}+\mathbf{K}\left(\mathrm{L}^{2}\left(M^{\omega}\right)\right)$ ) is a $\mathrm{C}^{*}$-subalgebra of $\mathbf{B}\left(\mathrm{L}^{2}(M)\right)$ (resp. $\left.\mathbf{B}\left(\mathrm{L}^{2}\left(M^{\omega}\right)\right)\right)$. Fix a faithful state $\varphi \in M_{*}$. Denote by $e: \mathrm{L}^{2}(M) \rightarrow \mathbf{C} \xi_{\varphi}$ and $f: \mathrm{L}^{2}\left(M^{\omega}\right) \rightarrow$ $\mathbf{C} \xi_{\varphi^{\omega}}$ the corresponding orthogonal projections. Observe that $\mathbf{K}\left(\mathrm{L}^{2}(M)\right)$ is the norm closure in $\mathbf{B}\left(\mathrm{L}^{2}(M)\right)$ of $M e M$. Denote by $N:=\prod^{\omega} M$ the Groh-Raynaud ultraproduct and by $p \in N$ the support projection of the ultraproduct state $\varphi_{\omega} \in N_{*}$.

Claim. There is a $*$-homomorphism $\theta: C+\mathbf{K}\left(\mathrm{L}^{2}(M)\right) \rightarrow \mathbf{B}\left(\mathrm{L}^{2}\left(M^{\omega}\right)\right)$ such that $\theta(x)=x$ and $\theta\left(J^{M} y J^{M}\right)=J^{M^{\omega}} y J^{M^{\omega}}$ for all $x, y \in M$ and $\theta(e)=f$.

Proof of the Claim. Keep the same notation as in the proof of the Claim of Proposition 3.4. By Lemma 2.2, $\pi^{\omega}\left((e)_{n}\right)$ commutes with $p$ and $J^{N}$ and hence $\pi^{\omega}\left((e)_{n}\right)$ commutes with $\widetilde{p}:=$ $p J^{N} p J^{N}$. Since $\widetilde{p}$ commutes with $\pi^{\omega}(M)$ and $\pi^{\omega}\left(J^{M} M J^{M}\right)=J^{N} \pi^{\omega}(M) J^{N}, \widetilde{p}$ commutes with $\pi^{\omega}\left(C+\mathbf{K}\left(\mathrm{L}^{2}(M)\right)\right)$. Recall that $\widetilde{p} N \widetilde{p} \cong p N p \cong M^{\omega}$ and $\widetilde{p} \mathrm{~L}^{2}(M)_{\omega}=\mathrm{L}^{2}\left(M^{\omega}\right)$. Then the *-homomorphism

$$
\theta: C+\mathbf{K}\left(\mathrm{L}^{2}(M)\right) \rightarrow \mathbf{B}\left(\mathrm{L}^{2}\left(M^{\omega}\right)\right): T \mapsto \widetilde{p} \pi^{\omega}(T) \widetilde{p}
$$

satisfies all the conditions of the Claim. 
Since $\theta(C)=C_{\omega}$ and $\theta\left(\mathbf{K}\left(\mathrm{L}^{2}(M)\right)\right) \subset \mathbf{K}\left(\mathrm{L}^{2}\left(M^{\omega}\right)\right), \theta$ induces a $*$-homomorphism

$$
\widetilde{\theta}:\left(C+\mathbf{K}\left(\mathrm{L}^{2}(M)\right)\right) / \mathbf{K}\left(\mathrm{L}^{2}(M)\right) \rightarrow\left(C_{\omega}+\mathbf{K}\left(\mathrm{L}^{2}\left(M^{\omega}\right)\right)\right) / \mathbf{K}\left(\mathrm{L}^{2}\left(M^{\omega}\right)\right) .
$$

Denote by $\iota: A \otimes_{\min } J^{M^{\omega}} B J^{M^{\omega}} \rightarrow A \otimes_{\min } J^{M} B J^{M}$ the tautological *-isomorphism. Then the composition map

$$
\nu_{\omega}=\tilde{\theta} \circ \nu \circ \iota: A \otimes_{\mathrm{alg}} J^{M^{\omega}} B J^{M^{\omega}} \rightarrow \mathbf{B}\left(\mathrm{L}^{2}\left(M^{\omega}\right)\right) / \mathbf{K}\left(\mathrm{L}^{2}\left(M^{\omega}\right)\right)
$$

is continuous with respect to the minimal tensor norm.

\section{REFERENCES}

[AO74] C.A. Akemann, P.A. Ostrand, On a tensor product $\mathrm{C}^{*}$-algebra associated with the free group on two generators. J. Math. Soc. Japan 27 (1975), 589-599.

[AH12] H. Ando, U. HAAgerup, Ultraproducts of von Neumann algebras. J. Funct. Anal. 266 (2014), 6842-6913.

[BdlHV08] M. BekKa, P. de la Harpe, A. Valette, Kazhdan's property (T). New Mathematical Monographs, 11. Cambridge University Press, Cambridge, 2008. xiv +472 pp.

[BISG15] R. Boutonnet, A. Ionna, A. SAlehi Golsefidy, Local spectral gap in simple Lie groups and applications. arXiv:1503.06473

[BN11] L. Bowen, A. Nevo, Pointwise ergodic theorems beyond amenable groups. Ergodic Theory Dynam. Systems 33 (2013), 777-820.

[BO08] N.P. Brown, N. Ozawa, $C^{*}$-algebras and finite-dimensional approximations. Graduate Studies in Mathematics, 88. American Mathematical Society, Providence, RI, 2008.

[Ch81] M. ChodA, Inner amenability and fullness. Proc. Amer. Math. Soc. 86 (1982), 663-666.

[Co72] A. Connes, Une classification des facteurs de type III. Ann. Sci. École Norm. Sup. 6 (1973), 133-252.

[Co74] A. Connes, Almost periodic states and factors of type $\mathrm{III}_{1}$. J. Funct. Anal. 16 (1974), 415-445.

[Co75] A. Connes, Classification of injective factors. Cases $\mathrm{II}_{1}, \mathrm{II}_{\infty}, \mathrm{III}_{\lambda}, \lambda \neq 1$. Ann. of Math. 74 (1976), $73-115$.

[Co85] A. Connes, Factors of type $\mathrm{III}_{1}$, property $L_{\lambda}^{\prime}$ and closure of inner automorphisms. J. Operator Theory 14 (1985), 189-211.

[CJ81] A. Connes, V.F.R. Jones, A $\mathrm{II}_{1}$ factor with two non-conjugate Cartan subalgebras. Bull. Amer. Math. Soc. 6 (1982), 211-212.

[CS78] A. Connes, E. Størmer, Homogeneity of the state space of factors of type $\mathrm{III}_{1}$. J. Funct. Anal. 28 (1978), 187-196.

[FM75] J. Feldman, C.C. Moore, Ergodic equivalence relations, cohomology, and von Neumann algebras. I, II. Trans. Amer. Math. Soc. 234 (1977), 289-324, 325-359.

[Ga10] D. Gaboriau, Orbit equivalence and measured group theory. Proceedings of the International Congress of Mathematicians (Hyderabad, 2010), Vol. III, Hindustan Book Agency (2010), 1501-1527.

[HS90] U. HaAgerup, E. StøRmer, Equivalence of normal states on von Neumann algebras and the flow of weights. Adv. Math. 83 (1990), 180-262.

[Ho15] D.J. HofF, Von Neumann algebras of equivalence relations with nontrivial one-cohomology. J. Funct. Anal. 270 (2016), 1501-1536.

[HI15] C. HoudAyer, Y. IsONo, Unique prime factorization and bicentralizer problem for a class of type III factors. arXiv: 1503.01388

[HR14] C. Houdayer, S. Raum, Asymptotic structure of free Araki-Woods factors. Math. Ann. 363 (2015), $237-267$.

[HU15] C. HoudAyER, Y. UEDA, Rigidity of free product von Neumann algebras. arXiv: 1507.02157

[HV12] C. Houdayer, S. Vaes, Type III factors with unique Cartan decomposition. J. Math. Pures Appl. 100 (2013), 564-590.

[Io12a] A. IoAnA, Cartan subalgebras of amalgamated free product $\mathrm{II}_{1}$ factors. Ann. Sci. École Norm. Sup. 48 (2015), 71-130.

[Io12b] A. IoAnA, Classification and rigidity for von Neumann algebras. Proceedings of the 6th European Congress of Mathematics (Krakow, 2012), European Mathematical Society Publishing House

[Is12] Y. Isono, Weak exactness for $\mathrm{C}^{*}$-algebras and application to condition (AO). J. Funct. Anal. 264 (2013), 964-998.

[Is13] Y. Isono, On bi-exactness of discrete quantum groups. Int. Math. Res. Not. Volume 2014, Article ID rnu043.

[MvN43] F. Murray, J. von Neumann, Rings of operators. IV. Ann. of Math. 44 (1943), 716-808. 
[Oc85] A. Ocneanu, Actions of discrete amenable groups on von Neumann algebras. Lecture Notes in Mathematics, 1138. Springer-Verlag, Berlin, 1985. iv+115 pp.

[Oz03] N. Ozawa, Solid von Neumann algebras. Acta Math. 192 (2004), 111-117.

[Oz04] N. Ozawa, A Kurosh type theorem for type $\mathrm{II}_{1}$ factors. Int. Math. Res. Not. (2006), Art. ID 97560, $21 \mathrm{pp}$.

[Oz16] N. OzawA, A remark on fullness of some group measure space von Neumann algebras. arXiv: 1602.02654

[Pe06] J. Peterson, L L -rigidity in von Neumann algebras. Invent. Math. 175 (2009), 417-433.

[Po95] S. PopA, Classification of subfactors and their endomorphisms. CBMS Regional Conference Series in Mathematics, 86. Published for the Conference Board of the Mathematical Sciences, Washington, DC; by the American Mathematical Society, Providence, RI, 1995. x+110 pp.

[Po01] S. PopA, On a class of type $\mathrm{II}_{1}$ factors with Betti numbers invariants. Ann. of Math. 163 (2006), 809-899.

[Po03] S. POPA, Strong rigidity of $\mathrm{II}_{1}$ factors arising from malleable actions of w-rigid groups $\mathrm{I}$. Invent. Math. 165 (2006), 369-408.

[Po06a] S. PopA, Deformation and rigidity for group actions and von Neumann algebras. Proceedings of the International Congress of Mathematicians (Madrid, 2006), Vol. I, European Mathematical Society Publishing House, 2007, p. 445-477.

[Po06b] S. Popa, On the superrigidity of malleable actions with spectral gap. J. Amer. Math. Soc. 21 (2008), 981-1000.

[Po06c] S. PopA, On Ozawa's property for free group factors. Int. Math. Res. Not. IMRN 2007, no. 11, Art. ID rnm036, $10 \mathrm{pp}$.

[PV11] S. POPA, S. VAES, Unique Cartan decomposition for $\mathrm{II}_{1}$ factors arising from arbitrary actions of free groups. Acta Math. 212 (2014), 141-198.

[Ra99] Y. RAYNAUd, On ultrapowers of non commutative $L_{p}$-spaces. J. Operator Theory 48 (2002), 41-68.

[Sa09] H. SAKo, Measure equivalence rigidity and bi-exactness of groups. J. Funct. Anal. 257 (2009), 31673202.

[Sh96] D. Shlyakhtenko, Free quasi-free states. Pacific J. Math. 177 (1997), 329-368.

[Sk88] G. SkAndalis, Une notion de nucléarité en K-théorie (d'après J. Cuntz). K-Theory 1 (1988), 549573. Encyclopaedia of Mathematical Sciences, 125. Operator Algebras and Non-commutative Geometry, 6. Springer-Verlag, Berlin, 2003. xxii+518 pp.

[TU14] R. Tomatsu, Y. UEdA, A characterization of fullness of continuous cores of type III $_{1}$ free product factors. To appear in Kyoto J. Math. arXiv:1412.2418

[Ue00] Y. UEDA, Fullness, Connes' x-groups, and ultra-products of amalgamated free products over Cartan subalgebras. Trans. Amer. Math. Soc. 355 (2003), 349-371.

[Va10] S. VAEs, Rigidity for von Neumann algebras and their invariants. Proceedings of the International Congress of Mathematicians (Hyderabad, 2010), Vol. III, Hindustan Book Agency, 2010, 1624-1650.

[VV05] S. VAEs, R. Vergnioux, The boundary of universal discrete quantum groups, exactness, and factoriality. Duke Math. J. 140 (2007), 35-84.

[Zi84] R. Zimmer, Ergodic theory and semisimple groups. Monographs in Mathematics, 81. Birkhäuser Verlag, Basel, 1984. x+209 pp.

Laboratoire de Mathématiques d’Orsay, Université Paris-Sud, CNRS, Université Paris-Saclay, 91405 Orsay, France

E-mail address: cyril.houdayer@math.u-psud.fr

RIMS, Kуото University, 606-8502 Күото, JAPAN

E-mail address: isono@kurims.kyoto-u.ac.jp 